\title{
DYNAMICS OF LINEAR AND AFFINE MAPS*
}

\author{
RAVI S. KULKARNI ${ }^{\dagger}$
}

\begin{abstract}
The well-known theory of the "rational canonical form of an operator" describes the invariant factors, or equivalently, elementary divisors, as a complete set of invariants of a similarity class of an operator on a finite-dimensional vector space $\mathbb{V}$ over a given field $\mathbb{F}$. A finer part of the theory is the contribution by Frobenius dealing with the structure of the centralizer of an operator. The viewpoint is that of finitely generated modules over a PID, cf. for example [8], ch. 3. In this paper we approach the issue from a "dynamic" viewpoint. We also extend the theory to affine maps. The formulation is in terms of the action of the general linear group $G L(n)$, resp. the group of invertible affine maps $G A(n)$, on the semigroup of all linear, resp. affine, maps by conjugacy. The theory of rational canonical forms is connected with the orbits, and the Frobenius' theory with the orbit-classes, of the action of $G L(n)$ on the semigroup of linear maps. We describe a parametrization of orbits and orbit-classes of both $G L(n)$ - and $G A(n)$-actions, and also provide a parametrization of all linear and affine maps themselves, which is independent of the choices of linear or affine coordinate systems, cf. sections 7, 8, 9. An important ingredient in these parametrizations is a certain flag. For a linear map $T$ on $\mathbb{V}$, let $Z_{L}(T)$ denote its centralizer associative $\mathbb{F}$-algebra, and $Z_{L}(T)^{*}$ the multiplicative group of invertible elements in $Z_{L}(T)$. In this situation, we associate a canonical, maximal, $Z_{L}(T)$-invariant flag, and precisely describe the orbits of $Z_{L}(T)^{*}$ on $\mathbb{V}$, cf. section 3 . The classical theory uses only invariance under $T$, i.e. $\mathbb{V}$ is considered only as a module over $\mathbb{F}[T]$. The finer invariance under $Z_{L}(T)$, i.e. considering $\mathbb{V}$ as a module over $Z_{L}(T)$, makes the construction of the flag canonical. We believe that this flag has not appeared before in this classical subject. Using this approach, we strengthen the classical theory in a number of ways.
\end{abstract}

Key words. Rational canonical form, centraliser, dynamical types

AMS subject classifications. 12E99, 15A01, 15A02, 15A21, 37C45

1. Introduction. Let $\mathbb{F}$ be a field, and $\mathbb{V}$ an $n$-dimensional vector space over $\mathbb{F}$. Let $L(\mathbb{V})$ denote the set of all linear maps from $\mathbb{V}$ to $\mathbb{V}$. Underlying $\mathbb{V}$ there is the affine space $\mathbb{A}$. Intuitively, $\mathbb{A}$ has no distinguished base-point which one can call as the "zero", or the "origin". However there is a well-defined notion of "difference of points". When we distinguish a base-point $O$, and call it the zero, then there is a well-defined notion of addition, making $\mathbb{A}$ into a vector space. An affine map of $\mathbb{A}$ is a map $(A, v): \mathbb{V} \rightarrow \mathbb{V}$ of the form $(A, v)(x)=A x+v$, where $A$ is in $L(\mathbb{V})$, and $x, b$ are in $\mathrm{V}$. Then

$$
\left(A_{1}, v_{1}\right) \circ\left(A_{2}, v_{2}\right)=\left(A_{1} \circ A_{2}, A_{1} v_{2}+v_{1}\right) .
$$

This formula shows that $A(\mathbb{V})$ is a semigroup with identity under composition, and $L(\mathbb{V})$ is a sub-semigroup of $A(\mathbb{V})$.

It is important to note that the representation $(A, v)$ depends on the choice of the base-point. However the semigroup of affine maps, and the form of an affine map is independent of this choice. Indeed, let $O$ be a base-point making $\mathbb{A}$ into a vector space $\mathbb{V}$. Let $P$ be another point of $\mathbb{A}$ with the associated vector $a$. Let $x$ resp $y$ be vector representations of a point $Q$ w.r.t. base-points $O$ and $P$. Then $y=x-a$. Let $f$ be an affine map of the form $(A, v)$ in the $x$-representation, and $f(Q)=R$. Then the $x$-representation of $R$ is $A x+v=A y+A a+v$. So the $y$-representation of $R$ is $A y+A a+v-a=A y+w$, where $w=(A-I) a+v$. Hence the $y$-representation of $f$ is $(A, w)$. The maps induced by the action of the group $(\mathbb{V},+)$ on $\mathbb{V}$, called the

\footnotetext{
*Received April 17, 2007; accepted for publication April 22, 2008.

†Indian Institute of Technology (Bombay), Powai, Mumbai 400076, India, \& Queens College and Graduate Center, City University of New York (punekulk@yahoo.com; kulkarni@math.iitb.ac.in).
} 
translations, have the form $\tau_{a}=(I, a)$. They form a subgroup $\mathbb{T}$, which is of course isomorphic to $\mathbb{V}$. The above calculation shows that the expression for $\tau_{a}: x \mapsto x+a$ remains the same no matter where we choose the base-point. In other words, " $a$ " in $\tau_{a}$ has a dynamic as well as affine meaning. When $A \neq I$, the same calculation shows that " $A$ " remains the same no matter where we take the base-point, but " $v$ " may change. In other words, even when $A \neq I$, the " $A$ " has a dynamic meaning, but " $v$ " does not. The formula (1.1) shows that we have a well-defined homomorphism $l: A(\mathbb{V}) \rightarrow L(\mathbb{V})$ given by $l((A, v))=A$. We shall call $A$ the linear part of $(A, v)$. We shall also call $v$ the translational part of $(A, v)$, with the understanding that this specification depends on the choice of the base-point. Note that the kernel of $l$, namely $l^{-1}(I)$ consists precisely of $\mathbb{T}$.

Let us also note an inconsistency in the usual terminology. Probably following the usage in the fields such as Transformation Groups, or Transformation Geometry, the phrase "an affine transformation" usually means a bijective affine map. On the other hand, in Linear Algebra, the phrase "a linear transformation" is used for nonbijective linear maps as well. To avoid confusion, and also for brevity, we use a neutral terminology "linear maps" or "affine maps" for not necessarily bijective maps.

We may also like to define

$$
\left(A_{1}, v_{1}\right)+\left(A_{2}, v_{2}\right)=\left(A_{1}+A_{2}, v_{1}+v_{2}\right) .
$$

As is well-known, $L(\mathbb{V})$ becomes an associative $F$-algebra with this definition of addition, and taking composition as multiplication. However, we note that with the same definitions, in $A(\mathbb{V})$, we do not get left distributivity of multiplication w.r.t addition. So $A(\mathbb{V})$ becomes only a "near ring", or better a "near $\mathbb{F}$-algebra", cf. for example, [10]. Let $G L(\mathbb{V})$, resp. $G A(\mathbb{V})$, denote the subsets of $L(\mathbb{V})$, resp $A(\mathbb{V})$ consisting of invertible elements. They form groups under composition, and $G L(\mathbb{V})$ is a subgroup of $G A(\mathbb{V})$. They act on $L(\mathbb{V})$ resp. $A(\mathbb{V})$ by conjugation. Namely $f$ in $G L(\mathbb{V})$, resp. $G A(\mathbb{V})$, acts on $L(\mathbb{V})$, resp. in $A(\mathbb{V})$ by $T \mapsto f T f^{-1}$. We denote these actions by $\phi_{L}$ resp $\phi_{A}$. When there will be no confusion, we shall also abbreviate them to $\phi$.

Our interest in this paper is to study the "dynamics" of $L(\mathbb{V})$ and $A(\mathbb{V})$. We interpret the words "study of dynamics" to mean

i) Parametrization of the $\phi$-orbits of $G L(\mathbb{V})$, resp. $G A(\mathbb{V})$, on $L(\mathbb{V})$, resp. $A(\mathbb{V})$, cf. theorem 7.1.

ii) In any action of a group $G$ on a set $X$ we have a notion of orbit-equivalence. Namely, $x, y$ in $X$ are orbit-equivalent iff the stabilizer subgroups $G_{x}$, and $G_{y}$ are conjugate, cf. [9], theorem 2.1 for a precise statement on the structure of an orbitequivalence class, as a certain set-theoretic fibration. In the case of the $\phi$-action a stabilizer subgroup at $T$ in $G L(\mathbb{V})$ resp. $G A(\mathbb{V})$ is precisely the centralizer of $T$ in $G L(\mathbb{V})$ resp. $G A(\mathbb{V})$. We denote this subgroup by $Z_{L}^{*}(T)$, resp. $Z_{A}^{*}(T)$. For short, we call the orbit-equivalence in either the linear or the affine case, the $z$-equivalence. In this paper one of our main aims is to parametrize the $z$-equivalence classes of linear or affine maps, cf. theorem 7.2.

iii) Parametrizations of linear, resp. affine, maps which depend only on $\mathbb{F}$ and $\operatorname{dim} \mathbb{V}=n$, and not on the choice of a linear resp. affine coordinate system, cf. theorem 7.3.

Interestingly, in this case $G L(\mathbb{V})$, resp. $G A(\mathbb{V})$, are also subsets of $L(\mathbb{V})$, resp. $A(\mathbb{V})$, so there is also a notion of centraliers of $T$ is $L(\mathbb{V})$, resp $A(\mathbb{V})$. We denote these centralizers by $Z_{L}(T)$, resp $Z_{A}(T)$. Then $Z_{L}(T)$ is an $\mathbb{F}$-subalgebra of $L(\mathbb{V})$, and 
$Z_{A}(T)$ is a sub-near- $\mathbb{F}$-algebra of $A(\mathbb{V})$. In fact, $Z_{L}^{*}(T)$, resp. $Z_{A}^{*}(T)$, are precisely the groups of invertible elements in $Z_{L}(T)$, resp $Z_{A}(T)$.

A basic notion of "equivalence of dynamics" in our case is the following. First, let $T_{i}$ be elements of $L\left(\mathbb{V}_{i}\right), \mathrm{i}=1,2$. We say that the $T_{i}$ 's are "dynamically equivalent" if there is a linear isomorphism $h: \mathbb{V}_{1} \rightarrow \mathbb{V}_{2}$ such that $h \circ T_{1}=T_{2} \circ h$. In this case we shall also say that the pairs $\left(\mathbb{V}_{i}, T_{i}\right), \mathrm{i}=1,2$, are dynamically equivalent. Similarly let $T_{i}$ be elements of $A\left(\mathbb{V}_{i}\right), \mathrm{i}=1,2$. We say that the $T_{i}$ 's are "dynamically equivalent" if there is an affine isomorphism $h: \mathbb{A}_{1} \rightarrow \mathbb{A}_{2}$ such that $h \circ T_{1}=T_{2} \circ h$.

Next, let $T$ be an element of $L(\mathbb{V})$. We say that $\mathbb{V}$ is $T$-decomposable, or the pair $(\mathbb{V}, T)$ is decomposable, or more loosely also that $T$ is decomposable, if $\mathbb{V}$ is a direct sum of proper $T$-invariant subspaces. Otherwise $\mathbb{V}$ is said to be $T$-indecomposable. Since $\operatorname{dim} \mathbb{V}$ is finite, clearly $\mathbb{V}$ is a direct sum of finitely many $T$-indecomposable invariant subspaces. Also the pair $(\mathbb{V}, T)$ is indecomposable iff any dynamically equivalent pair is indecomposable. So from a dynamic viewpoint, a basic problem is to describe suitable models of indecomposable $(\mathbb{V}, T)$ 's, and secondly, given a pair $(\mathbb{V}, T)$ to understand in general all decompositions of $\mathbb{V}$ into $T$-indecomposable subspaces. The first problem is solved by the theory of "rational canonical form of a square matrix" as a special case of modules over principal ideal domains. In this classical approach, the second problem gets obscured in a clever inductive proof. Following a dynamic viewpoint, we shall offer a new view of both the problems which, in some sense, is "dual" to the the classical approach.

This approach strengthens the classical theory in a number of ways. In this paper we have considered the following aspects.

i) Making an essential use of the $Z_{L}(T)$-invariant flag, we determine the conjugacy classes, the centralizers, and $z$-classes of both linear and affine maps. The consideration of affine maps naturally arises in the study of affine ODEs (where $\mathbb{F}=\mathbb{R}$ ), cf. section 6. The texts of ODEs appeal to a general "method of variation of parameters". In our opinion, the dynamic approach offers a better insight. At the same time, we are not aware of any literature on the general case, from the viewpoint of $G A(\mathbb{V})$-action on $A(\mathbb{V})$.

ii) We derive a necessary and sufficient condition for the existence of "S $+\mathrm{N}$ "decomposition of an operator - or its multiplicative analogue, the "SU"-decomposition of an invertible operator - and its relation to lifts of $\mathbb{E}$-structures, cf. section 5. A basic observation going back to Maurer in special cases, cf. [1], [2], [4], is that a linear algebraic group over a field of characteristic 0 , contains the semisimple and unipotent parts of each of its elements. If the base-field is of positive characteristic such decomposition in general does not exist. In this context one introduces the notion of perfectness of the base-field, which is a sufficient, but not necessary, condition for the existence of such decomposition. The dynamic viewpoint provides an overall insight on this ticklishly confusing point in the theory of linear algebraic groups.

iii) We derive a dynamic interpretation of Frobenius' "double commutant" theorem, cf, section 4.

iv) We prove the following finiteness result: If $\mathbb{F}$ has the property that there are only finitely many field-extensions of $\mathbb{F}$ of degrees at most $n$, then there are only finitely many $z$-classes in $L(\mathbb{V})$, and $A(\mathbb{V})$. For example, if $\mathbb{F}$ is an algebraically closed field, a real closed field, or a local field then $\mathbb{F}$ has the stated property. This is a major example which illustrates the viewpoint that motivated [9]. In the forthcoming papers we hope to extend this work to transformations in other classical geometries.

v) We obtain the generating functions for $z$-classes of linear maps, in some cases 
when there are only finitely many such $z$-classes in each dimension. They are related to the generating function for partitions in an interesting way. They appear to be a new type of generating functions which have not appeared in number theory before. We only make some elementary observations regarding these generating functions.

In some sense, the dynamical viewpoint has brought together two seemingly separate chapters of Linear Algebra, namely, i) "rational canonical forms", and ii) "Frobenius's theory of centralisers", of operators on a finite-dimensional vector spaces.

Before closing this introduction, we would like to remark that from a dynamic viewpoint, the minimal polynomial $m_{T}(x)$ is perhaps a more basic invariant than the more easily computable invariant $\chi_{T}(x)$, the characteristic polynomial of $T$. One indication of this fact is that $m_{T}(x)$ is defined even when $\mathbb{V}$ is infinite-dimensional. Most results of this paper can be suitably extended to the case when $\mathbb{V}$ is infinitedimensional, and $m_{T}(x) \neq 0$. However, to keep a focus we do not elaborate on this direction here, as we had done in [9].

I wish to acknowledge the benefit of many conversations on the contents of this work with Rony Gouraige. His thesis, cf. [6], (City University of New York, 2006) partially extends this work to operators on finite-dimensional vector spaces over skewfields. It is also a pleasure to acknowledge some conversations with I. B. S. Passi, and Surya Ramana at the Harish-Chandra Research Institute, Allahabad, India, regarding the "S + N"-decomposition.

2. Classical Theory for $L(\mathbb{V})$. Let $m_{T}(x)$ denote the minimal polynomial of $T$. If $\mathbb{F}[T]$ denotes the $\mathbb{F}$-algebra generated by $T$, then $F[T] \approx F[x] /\left(m_{T}(x)\right)$. Let $m_{T}(x)=\Pi_{i=1}^{r} p_{i}(x)^{d_{i}}$ be the decomposition into irreducible factors. Here $p_{i}(x)$ is a monic irreducible polynomial in $\mathbb{F}[x]$, and $p_{i}(x)$ 's are pairwise distinct. We shall call $p_{i}(x)$ 's the primes associated to $T$. The first step in the theory provides a decomposition $\mathbb{V}=\oplus_{i=1}^{r} \mathbb{V}_{i}$ into $T$-invariant subspaces. Here $\mathbb{V}_{i}=\operatorname{ker} p_{i}(T)^{d_{i}}$. We observe that this decomposition is in fact invariant under $Z_{L}(T)$, the $\mathbb{F}$-subalgebra of $L(\mathbb{V})$ consisting of all operators commuting with $\mathbb{T}$. Let $T_{i}$ denote the restriction of $T$ to $\mathbb{V}_{i}$. Then $m_{T_{i}}(x)=p_{i}(x)^{d_{i}}$. Moreover we have a canonical $\mathbb{F}$-algebra decomposition.

$$
Z_{L}(T)=\Pi_{i=1}^{r} Z_{L}\left(T_{i}\right)
$$

So to describe the indecomposable pairs $(\mathbb{V}, T)$ we have reduced to the situation where $m_{T}(x)=p(x)^{d}$, where $p(x)$ is a monic irreducible polynomial in $\mathbb{F}[x]$.

At this point, we note a crucial example. Consider the algebra $\mathbb{V}=\mathbb{F}[x] /\left(p(x)^{d}\right)$, but consider it only as an $\mathbb{F}$-vector space. For $u(x)$ in $\mathbb{F}[x]$ let $[u(x)]$ denote the class of $u(x)$ in $\mathbb{F}[x] /\left(p(x)^{d}\right)$. Let $T=\mu_{x}$ be the operator $[u(x)] \mapsto[x u(x)]$. For $i=0,1, \ldots, d$, let $\mathbb{V}_{i}=\left\{\left[f(x) p(x)^{i}\right] \mid f(x) \in \mathbb{F}[x]\right\}$. Clearly we have a flag of subspaces

$$
0=\mathbb{V}_{d} \subset \mathbb{V}_{d-1} \ldots \subset \mathbb{V}_{1} \subset \mathbb{V}_{0}=\mathbb{V}
$$

The claim is that $\mathbb{V}_{i}$ 's are precisely all the $T$-invariant subspaces of $\mathbb{V}$. Indeed let $\mathbb{W}$ be a $T$-invariant subspace of $\mathbb{V}$. If $\left[f(x) p(x)^{i}\right]$ is in $\mathbb{W}$, then by $T$-invariance, for all $g(x)$ in $\mathbb{F}[x]$, we have $\left[g(x) f(x) p(x)^{i}\right]$ also in $\mathbb{W}$. Let $i$ be the least non-negative integer such that $\mathbb{W}$ contains an element of the form $\left[f(x) p(x)^{i}\right]$ such that $p(x)$ does not divide $f(x)$. Then $[f(x)]$ is a unit in the algebra $\mathbb{F}[x] /\left(p(x)^{d}\right)$. So $\left[p(x)^{i}\right]$ is in $\mathbb{W}$. It easily follows that $\mathbb{W}=\mathbb{V}_{i}$. Notice that no $\mathbb{V}_{i}$ has a proper complementary subspace. So $(V, T)$ is an indecomposable pair.

For the future, notice that in this case $\operatorname{dim}_{\mathbb{F}} \mathbb{V}=\operatorname{deg} p(x)^{d}=\operatorname{d} \operatorname{deg} p(x)$. 
A second and major step in the theory is that the converse of the observation in the above example is true.

Theorem 2.1. Let $(\mathbb{V}, T)$ be an indecomposable pair. Then it is dynamically equivalent to $\left(\mathbb{F}[x] /\left(p(x)^{d}\right), \mu_{x}\right)$, for some monic irreducible polynomial $p(x)$ in $\mathbb{F}[x]$.

In view of the reduction in the first step, clearly an equivalent statement is the following.

THEOREM 2.2. Let $(\mathbb{V}, T)$ be a pair such that $m_{T}(x)=p(x)^{d}$, where $p(x)$ is a monic irreducible polynomial $p(x)$ in $\mathbb{F}[x]$, of degree $m$. Then $(\mathbb{V}, T)$ is a direct sum of T-invariant indecomposable subspaces, each dynamically equivalent to $\left(\mathbb{F}\left[x_{i}\right] /\left(p\left(x_{i}\right)^{d_{i}}\right), \mu_{x_{i}}\right)$. Here $d_{i} \leq d$, for at least one $i$, we have $d_{i}=d$, and $\operatorname{dim} \mathbb{V}=m \sum_{i} d_{i}$.

We note that in case $d=1$, the proof of either of these statements is easier than in the classical approach dealing with the more general situation of finitely generated modules over a PID. We sketch it first. The general case $d \geq 2$ will be considered in the next section.

Indeed observe that $\mathbb{E}=\mathbb{F}[x] /(p(x))$ w.r.t. to its standard additive and multiplicative structures is a field. In fact it is a simple field extension of $\mathbb{F}$. Here "simple" means that $\mathbb{E}$ is generated over $\mathbb{F}$ by a single element $[x]$. Indeed $[x]$ is a root of $p(x)$ in $\mathbb{E}$, and in the language of field theory $[x]$ is a primitive element of $\mathbb{E}$ over $\mathbb{F}$. Thus the operation of $T$ on $\mathbb{V}$, which amounts to multiplication by $[x]$, or $\left[x_{i}\right]$ 's in the standard models $\left(\mathbb{F}\left[x_{i}\right] /\left(p\left(x_{i}\right)\right), \mu_{x_{i}}\right)$, equips $\mathbb{V}$ with the structure of a vector space over $\mathbb{E}$, which extends its structure as a vector space over $\mathbb{F}$. In this $\mathbb{E}$-structure, the $T$-invariant subspaces are precisely the $\mathbb{E}$-subspaces of $\mathbb{V}$. Also an $\mathbb{F}$-linear operator $S$ is in $Z_{L}(T)$ iff $S$ is an $\mathbb{E}$-linear operator. It follows that $(\mathbb{V}, T)$ is indecomposable iff $\operatorname{dim}_{\mathbb{E}} \mathbb{V}=1$. Equivalently, $(\mathbb{V}, T)$ is decomposable iff $\operatorname{dim}_{\mathbb{E}} \mathbb{V}=r \geq 2$. In this case, a choice of an $\mathbb{E}$-basis leads to a $T$-invariant decomposition of $\mathbb{V}$ into $T$-indecomposable subspaces. The ambiguity in the choice of a $T$-invariant decomposition of $\mathbb{V}$ is precisely the ambiguity of choosing an $\mathbb{E}$-basis. Here $Z_{L}(T) \approx L_{\mathbb{E}}(\mathbb{V})$, and $Z_{L}(T)^{*} \approx G L_{\mathbb{E}}(\mathbb{V})$. The orbits of $Z_{L}(T)^{*}$ on $\mathbb{V}$ are $\{0\}$, and $\mathbb{V}-\{0\}$. As a module over the associative F-algebra $Z_{L}(T)$ or the group $Z_{L}(T)^{*}, \mathbb{V}$ is irreducible. Moreover the $T$-action is $d y$ namically semi-simple in the sense that every $T$-invariant subspace has a $T$-invariant complement.

A word of caution regarding the use of the phrase "dynamically semi-simple". There is another notion of semi-simplicity: namely, $T$ is algebraically semi-simple if it is diagonalizable on $\mathbb{V} \otimes \tilde{\mathbb{F}}$, where $\tilde{\mathbb{F}}$ denotes the algebraic closure of $\mathbb{F}$, cf. [1]. Contrary to some mis-statements in the literature the notions of algebraic semi-simplicity and dynamic semi-simplicity are not equivalent. They differ when the characteristic of $\mathbb{F}$ is not 0 , and $\mathbb{F}$ is not perfect. See section 5 .

Now note that as an associative $\mathbb{E}$-algebra, $Z_{L}(T)$ is simple, and $\mathbb{E}$ can be recovered from $Z_{L}(T)$ as its center. Since $\mathbb{E}$ is a simple field extension, we have also verified Frobenius's well-known "bi-commutant theorem", in the special case $d=1$, namely an operator which commutes with every operator which commutes with $T$ is a polynomial in $T$.

The case $d \geq 2$ is much more difficult. In this case the dynamical approach provides a different, in some sense "dual", insight, over the classical theory. We turn to this case in the next section. 
3. Orbits of $Z_{L}(T)^{*}$, and a Canonical Maximal $Z_{L}(T)$-invariant Flag. Let $T \in L(\mathbb{V}), m_{T}(x)=p(x)^{d}$, where $p(x)$ is a monic irreducible polynomial $p(x)$ in $\mathbb{F}[x]$. Let $\operatorname{deg} p(x)=m$. So $\mathbb{E}=\mathbb{F}[x] /(p(x))$ is a simple field extension of $F$, and $\operatorname{dim}_{\mathbb{F}} \mathbb{E}=m$. Assume $d \geq 2$. Let $N=p(T)$, and $\mathbb{V}_{i}=k e r N^{i}, i=0,1,2, \ldots d$. Thus we have a $Z_{L}(T)$-invariant flag of subspaces

$$
0=\mathbb{V}_{0} \subset \mathbb{V}_{1} \subset \mathbb{V}_{2} \subset \ldots \subset \mathbb{V}_{d}=\mathbb{V}
$$

We note an immediate consequence. Let $\bar{T}_{i}$ denote the operator induced by $T$ on $\mathbb{V}_{i} / \mathbb{V}_{i-1}, i=1,2, \ldots d$. Then $m_{\bar{T}_{i}}(x)=p(x)$. So by the case $d=1$ treated in the previous section we see that $\mathbb{V}_{i} / \mathbb{V}_{i-1}$ has a canonical $\mathbb{E}$-structure. So $\operatorname{dim}_{\mathbb{F}} \mathbb{V}_{i} / \mathbb{V}_{i-1}$, and finally $\operatorname{dim}_{\mathbb{F}} \mathbb{V}$ is divisible by $m$. So let $n=\operatorname{dim} \mathbb{V}=m l$.

We shall obtain a canonical, maximal $Z_{L}(T)$-invariant refinement of this flag. It will be convenient to use a double-subscript notation $\mathbb{V}_{i, j}$ for the subspaces occurring in this refined flag, with the understanding that $\mathbb{V}_{i}=\mathbb{V}_{i, 0}$. If we insert $k-1$ new terms between $\mathbb{V}_{i}$ and $\mathbb{V}_{i+1}$, we shall also denote $\mathbb{V}_{i+1}$ by $\mathbb{V}_{i, k}$. Our basic observation is: $\mathbb{V}_{i, j}-\mathbb{V}_{i, j-1}$ are precisely the $Z_{L}(T)^{*}$-orbits on $\mathbb{V}$. In particular, $\mathbb{V}_{i, j} / \mathbb{V}_{i, j-1}$ are irreducible, when they are considered as modules either over the group $Z_{L}(T)^{*}$ or over the $\mathbb{F}$-algebra $Z_{L}(T)$. Let $\bar{T}_{i, j}$ denote the operator induced by $T$ on $\mathbb{V}_{i, j} / \mathbb{V}_{i, j-1}$. It will turn out that $m_{\bar{T}_{i, j}}(x)=p(x)$. So by the case $d=1$ discussed in the last section, we have a canonical $\mathbb{E}$-structure on $\mathbb{V}_{i, j} / \mathbb{V}_{i, j-1}$. Let $\sigma=\operatorname{dim}_{\mathbb{E}} \mathbb{V}_{i, j} / \mathbb{V}_{i, j-1}$, and let $\mathbb{W}_{\sigma}$ denote an (abstract) vector space of dimension $\sigma$ over $\mathbb{E}$. As it will turn out, the algebra of operators induced by $Z_{L}(T)$ on $\mathbb{V}_{i, j} / \mathbb{V}_{i, j-1}$ is dynamically equivalent to the standard action of $L_{\mathbb{E}}\left(\mathbb{W}_{\sigma}\right)$ on $\mathbb{W}_{\sigma}$.

Before running into the proofs of these assertions, for the convenience of the reader, let us reconcile, albeit partially, this description with the classical theory. The classical theory attaches to $T$ as above, its elementary divisors, which are polynomials of the form $p(x)^{s_{i}}, i=1,2, \ldots r$. We may assume that $1 \leq s_{1}<s_{2}<\ldots<s_{r}=d$ are the distinct exponents of these elementary divisors, and $\sigma_{i}$ is the multiplicity of $p(x)^{s_{i}}$. Then $l=\Sigma_{i=1}^{r} s_{i} \sigma_{i}$, where $n=\operatorname{dim} \mathbb{V}=m l, m=\operatorname{deg} p(x)$. According to the classical theory the pair $(\mathbb{V}, T)$ is dynamically equivalent to the direct sum of the pairs of the form $\left(\mathbb{F}[x] /\left(p(x)^{s}, \mu_{x}\right)\right.$ where $s=s_{i}$ occurs $\sigma_{i}$ times, $i=1,2, \ldots, r$. It will turn out that the dimensions $\sigma$ of the (abstract) $\mathbb{E}$-vector spaces $\mathbb{W}_{\sigma}$ mentioned in the previous paragraph are precisely the multiplicities $\sigma_{i}$ 's of the elementary divisors in the classical theory. The refined flag mentioned above will independently pick up the exponents $s_{i}$ 's and multiplicities $\sigma_{i}^{\prime}$ s of the elementary divisors, subject to the relations, $l=\sum_{i=1}^{r} s_{i} \sigma_{i}$, where $n=\operatorname{dim} \mathbb{V}=m l, m=\operatorname{deg} p(x)$.

Let us now start building the refined flag. We shall first describe the refined flag where the dimensions of the subspaces in the flag are non-decreasing, and then offer a second description where these dimensions are strictly increasing.

Lemma 3.1. i) For $i>0, N=p(T)$ maps $\mathbb{V}_{i}$ into $\mathbb{V}_{i-1}$, and

ii) For $i>1$ the map induced by $N$ on $\mathbb{V}_{i} / \mathbb{V}_{i-1} \rightarrow \mathbb{V}_{i-1} / \mathbb{V}_{i-2}$ is injective.

The proof is straightforward, and is omitted.

Let $\left(e_{1}, e_{2}, \ldots e_{k}\right)$ be elements in $\mathbb{V}_{d}$ whose images $\left(\bar{e}_{1}, \bar{e}_{2}, \ldots \bar{e}_{k}\right)$ form an $\mathbb{E}$-basis of $\mathbb{V}_{d} / \mathbb{V}_{d-1}$. Then $T^{j}\left(e_{i}\right), 1 \leq j \leq m-1,1 \leq i \leq k$ are linearly independent over $\mathbb{F}$, as they are indepenedent over $\mathbb{F} \bmod \mathbb{V}_{d-1}$. Let $\mathbb{W}_{d}$ denote the $\mathbb{F}$-span of $T^{j}\left(e_{i}\right)$. Notice that by construction, $\mathbb{V}=\mathbb{V}_{d}=\mathbb{V}_{d-1}+\mathbb{W}_{d}$ is a direct sum of subspaces. Among these, $\mathbb{V}_{d-1}$ is $T$-invariant, but $\mathbb{W}_{d}$ is not (since we have assumed $d \geq 2$ ). 
However by construction, mod $\mathbb{V}_{d-1}$ it is $T$-invariant. We shall call such subspace of $\mathbb{V}_{d}$ an almost $T$-invariant subspace. Now notice that $N$ maps $\mathbb{W}_{d}$ injectively in $\mathbb{V}_{d-1}$ as a subspace complementary to $\mathbb{V}_{d-2}$. Moreover it is easy to check that $\mathbb{V}_{d-2}+N\left(\mathbb{W}_{d}\right)$ is independent of the choice of $\mathbb{W}_{d}$. It is a $T$-invariant, in fact $Z_{L}(T)$ invariant, subspace of $\mathbb{V}_{d-1}$. In case $\mathbb{V}_{d-2}+N\left(\mathbb{W}_{d}\right)$ is a proper subspace of $\mathbb{V}_{d-1}$ we insert it as an additional subspace in the flag between $\mathbb{V}_{d-2}$ and $\mathbb{V}_{d-1}$. Notice that $\left(\mathbb{V}_{d-2}+N\left(\mathbb{W}_{d}\right)\right) / \mathbb{V}_{d-2}$ is an $\mathbb{E}$-subspace of $\mathbb{V}_{d-1} / \mathbb{V}_{d-2}$.

Assume that $\mathbb{V}_{d-2}+N\left(\mathbb{W}_{d}\right)$ is a proper subspace of $\mathbb{V}_{d-1}$. For convenience, denote $k=\operatorname{dim}_{\mathbb{E}} \mathbb{V}_{d} / \mathbb{V}_{d-1}$ by $k_{d}$, and $e_{i}$ by $e_{d, i}$. Let $k_{d-1}=\operatorname{dim}_{\mathbb{E}} \mathbb{V}_{d-1} / \mathbb{V}_{d-2}-$ $\operatorname{dim}_{\mathbb{E}}\left(\mathbb{V}_{d-2}+N\left(\mathbb{W}_{d}\right)\right) / \mathbb{V}_{d-2}$. If $k_{d-1} \neq 0$, choose $e_{d-1, i}, 1 \leq i \leq k_{d-1}$ in $\mathbb{V}_{d-1}$ so that their classes mod $\mathbb{V}_{d-2}$ form an $\mathbb{E}$-basis of a subspace of $\mathbb{V}_{d-1} / \mathbb{V}_{d-2}$ complementary to $\left(\mathbb{V}_{d-2}+N\left(\mathbb{W}_{d}\right)\right) / \mathbb{V}_{d-2}$. Then $T^{j}\left(e_{d-1, i}\right), 1 \leq j \leq m-1,1 \leq i \leq k_{d-1}$ are clearly linearly independent over $\mathbb{F}$. Let $\mathbb{W}_{d-1}$ denote the $\mathbb{F}$-span of $T^{j}\left(e_{d-1, i}\right)$ 's. Then $\mathbb{W}_{d-1}$ is an almost $T$-invariant subspace of $\mathbb{V}_{d-1}$. Then $N$ maps $\mathbb{W}_{d-1}$ injectively into $\mathbb{V}_{d-2}$ onto a subspace complementary to $\mathbb{V}_{d-3}+N^{2}\left(\mathbb{W}_{d}\right)$. If $\mathbb{V}_{d-3}+N^{2}\left(\mathbb{W}_{d}\right)+N\left(\mathbb{W}_{d-1}\right)$ is a proper subspace of $\mathbb{V}_{d-2}$ we insert it as an additional subspace in the flag between $\mathbb{V}_{d-3}+N^{2}\left(\mathbb{W}_{d}\right)$ and $\mathbb{V}_{d-2}$. We note again that two subspaces $\mathbb{V}_{d-3}+N^{2}\left(\mathbb{W}_{d}\right)$ and $\mathbb{V}_{d-3}+N^{2}\left(\mathbb{W}_{d}\right)+N\left(\mathbb{W}_{d-1}\right)$ are independent of the choices of $\mathbb{W}_{d}$ and $\mathbb{W}_{d-1}$, and they are $Z_{L}(T)$-invariant subspaces of $\mathbb{V}_{d-2}$. In case $\mathbb{V}_{d-2}+N\left(\mathbb{W}_{d}\right)$ is not a proper subspace of $\mathbb{V}_{d-1}$, we simply take $\mathbb{W}_{d-1}$ to be 0 , and continue.

Proceeding in this way we obtain the following refined flag, where the dimension of the subspaces are non-decreasing.

$$
\begin{gathered}
0=\mathbb{V}_{0} \subset N^{d-1}\left(\mathbb{W}_{d}\right) \subset N^{d-1}\left(\mathbb{W}_{d}\right)+N^{d-2}\left(\mathbb{W}_{d-1}\right) \subset \ldots \\
N^{d-1}\left(\mathbb{W}_{d}\right)+N^{d-2}\left(\mathbb{W}_{d-1}\right)+\ldots N\left(\mathbb{W}_{2}\right)+\mathbb{W}_{1}=\mathbb{V}_{1} \subset \\
\mathbb{V}_{1}+N^{d-2}\left(\mathbb{W}_{d}\right) \subset \mathbb{V}_{1}+N^{d-2}\left(\mathbb{W}_{d}\right)+N^{d-3}\left(\mathbb{W}_{d-1}\right) \subset \ldots \\
\mathbb{V}_{1}+N^{d-2}\left(\mathbb{W}_{d}\right)+N^{d-3}\left(\mathbb{W}_{d-1}\right)+\ldots N\left(\mathbb{W}_{3}\right)+\mathbb{W}_{2}=\mathbb{V}_{2} \subset \ldots \\
\mathbb{V}_{d-3} \subset \mathbb{V}_{d-3}+N^{2}\left(\mathbb{W}_{d}\right) \subset \mathbb{V}_{d-3}+N^{2}\left(\mathbb{W}_{d}\right)+N\left(\mathbb{W}_{d-1}\right) \subset \\
\mathbb{V}_{d-3}+N^{2}\left(\mathbb{W}_{d}\right)+N\left(\mathbb{W}_{d-1}\right)+\mathbb{W}_{d-2}=\mathbb{V}_{d-2} \subset \\
\mathbb{V}_{d-2}+N\left(\mathbb{W}_{d}\right) \subset \mathbb{V}_{d-2}+N\left(\mathbb{W}_{d}\right)+\mathbb{W}_{d-1}=\mathbb{V}_{d-1} \subset \mathbb{V}_{d-1}+\mathbb{W}_{d}=\mathbb{V}_{d} .
\end{gathered}
$$


Notice that in this flag the sum $\oplus_{j=0}^{d-1} N^{j}\left(\mathbb{W}_{d}\right)$ forms a $T$-invariant (but not $Z_{L}(T)$ invariant) subspace dynamically equivalent to $k_{d}$ copies of $\mathbb{F}[x] /\left(p(x)^{d}\right)$. More generally the sums $\oplus_{j=0}^{s-1} N^{j}\left(\mathbb{W}_{s}\right), s=1,2, \ldots, d$ form a $T$-invariant (but not $Z_{L}(T)$ invariant) subspace dynamically equivalent to $k_{s}$ copies of $\mathbb{F}[x] /\left(p(x)^{s}\right)$, where $m k_{s}=$ $\operatorname{dim} \mathbb{W}_{s}$. If $\mathbb{W}_{s}=0$, then those terms effectively do not occur. By construction, $\mathbb{W}_{s}$ is the $\mathbb{F}$-span of $T^{j} e_{s, 1}, \ldots T^{j} e_{s, k_{s}}, 0 \leq j \leq m-1$. So $N^{u} T^{j} e_{s, 1}, \ldots N^{u} T^{j} e_{s, k_{s}}, 0 \leq j \leq$ $m-1,0 \leq u \leq s-1$ is a basis of $\oplus_{j=0}^{s-1} N^{j}\left(\mathbb{W}_{s}\right)$.

To get an irredundant flag where the dimesions are strictly increasing we need to proceed as follows. Let $1 \leq s_{1}<s_{2}<\ldots<s_{r}=d$ be integers such that $\mathbb{W}_{s_{i}} \neq 0$. Let $m \sigma_{i}=\operatorname{dim} W_{s_{i}}, 1 \leq i \leq r$. Let $\mathbb{V}_{i}=\mathbb{V}_{i, 0}$, and for $0 \leq i \leq s_{r-j+1}, 1 \leq j \leq r$, set

$$
\mathbb{V}_{i, j}=\mathbb{V}_{i}+N^{s_{r}-i}\left(\mathbb{W}_{s_{r}}\right)+N^{s_{r-1}-i}\left(\mathbb{W}_{s_{r-1}}\right)+\ldots+N^{s_{r-j+1}-i}\left(\mathbb{W}_{s_{r-j+1}}\right)
$$

A final important observation deals with the ambiguities involved in the choices of $\mathbb{W}_{s}$ where $s$ is one of the $s_{i}^{\prime}$ s. Notice that by construction, $\mathbb{W}_{s}$ is the $\mathbb{F}$-span of $T^{j} e_{s, 1}, \ldots T^{j} e_{s, k_{s}}, 0 \leq j \leq m-1$. So $N^{u} T^{j} e_{s, 1}, \ldots N^{u} T^{j} e_{s, k_{s}}, 0 \leq j \leq m-1,0 \leq$ $u \leq s-1$ is a basis of $\oplus_{j=0}^{s-1} N^{j}\left(\mathbb{W}_{s}\right)$. Let $\mathbb{W}_{s}^{\prime}$ be another choice of almost T-invariant subspace complementary to the subspace previous to $\mathbb{V}_{s+1}$ in the refined flag. Suppose $\mathbb{W}_{s}^{\prime}$ is constructed starting with $e_{s, 1}^{\prime}, e_{s, 2}^{\prime}, \ldots, e_{s, k_{s}}^{\prime}$. Let $N^{u} T^{j} e_{s, 1}^{\prime}, \ldots N^{u} T^{j} e_{s, k_{s}}^{\prime}, 0 \leq$ $j \leq m-1,0 \leq u \leq s-1$ be the corresponding basis of $\oplus_{j=0}^{s-1} N^{j}\left(\mathbb{W}_{s}^{\prime}\right)$. Then consider the $\mathbb{F}$-linear map which sends $N^{u} T^{j} e_{s, v}$ to $N^{u} T^{j} e_{s, v}^{\prime}$ and which is identity on the remaining $\oplus_{j=0}^{t-1} N^{j}\left(\mathbb{W}_{t}\right)$ for $t \neq s$. Clearly this map is invertible, commutes with $T$, and carries $\mathbb{W}_{s}$ into $\mathbb{W}_{s}^{\prime}$. In particular, repeating this argument to any two successive terms $\mathbb{V}_{i, j}$ and $\mathbb{V}_{i, j+1}$ we see that $Z_{L}(T)^{*}$ is transitive on $\mathbb{V}_{i, j+1}-\mathbb{V}_{i, j}$. In particular, this implies that $\mathbb{V}_{i, j+1} / \mathbb{V}_{i, j}$ is irreducible as a module over the group $Z_{L}(T)^{*}$ or the associative algebra $Z_{L}(T)$.

In particular, this completes the proof of theorem (2.1), or as noted earlier, equivalently, of theorem $(2,2)$. In the process however, we have strengthened the result which we record in the following form.

THEOREM 3.2. Let $T$ be in $E(\mathbb{V}), m_{T}(x)=p(x)^{d}$, where $p(x)$ is a monic irreducible polynomial in $\mathbb{F}[x]$. Then $\mathbb{V}$ admits a canonical, maximal $Z_{L}(T)$-invariant flag. A complement of each term appearing in the flag in its succeeding term is an orbit of $Z_{L}(T)^{*}$. In particular the quotient of each term appearing in the flag by its preceding term is an irreducible module over the group $Z_{L}(T)^{*}$, or the $\mathbb{F}$-algebra $Z_{L}(T)$.

As a comparison with the classical approach via modules over PID, we note that classically we regard $\mathbb{V}$ only as a module over $\mathbb{F}[T]$, and obtain the "vertical" decomposition of $\mathbb{V}$ whose summands are isomorphic to $\left(\mathbb{F}\left[x_{i}\right] /\left(p\left(x_{i}\right)^{d_{i}}\right), \mu_{x_{i}}\right)$. There is a wide choice in choosing these isomorphisms. They are not canonical. In the dynamical approach, we consider $\mathbb{V}$ as a module over $Z_{L}(T)$, and obtain $\mathbb{V}$ as an increasing union of a canonical, "horizontal", maximal, $Z_{L}(T)$-invariant flag. In this sense, we have called the dynamical approach giving a "dual" perspective vis-a-vis the classical approach.

As a by-product of this proof, we have some interesting dimension-counts, which refine the dimension counts in the well known Frobenuis' dimension formula. For simplicity, let $f(x)=p(x)^{d}$, where $p(x)$ is a monic irreducible polynomial in $\mathbb{F}[x], n=$ $\operatorname{dim} \mathbb{V}, m=\operatorname{deg} p(x)$, and $n=m l$. Consider a partition of $l$, namely, $l=\sum_{i=1}^{r} s_{i} \sigma_{i}$, 
where $s_{i}$ occurs $\sigma_{i}$ times, and we have assumed $1 \leq s_{1}<s_{2}<\ldots<s_{r}=d$. This data uniquely determines a pair $(\mathbb{V}, T)$ up to dynamical equivalence, with $m_{T}(x)=f(x)$.

To start with, we note again

- $n=\operatorname{dim} \mathbb{V}=m\left(s_{r} \sigma_{r}+s_{r-1} \sigma_{r-1}+\ldots+s_{1} \sigma_{1}.\right)$

- The successive sub-quotients associated to the flag, starting from $\mathbb{V}_{0}=0$, which are the Jordan constituents of $\mathbb{V}$ considered as a module over the associative algebra $Z_{L}(T)$, have $\mathbb{F}$-dimensions

$\left(m \sigma_{r}, m \sigma_{r-1}, \ldots, m \sigma_{1}\right)$ occurring $s_{1}$ times,

$\left(m \sigma_{r}, m \sigma_{r-1}, \ldots, m \sigma_{2}\right)$, occurring $s_{2}-s_{1}$ times,

..

$\left(m \sigma_{r}, m \sigma_{r-1}\right)$ occurring $s_{r-1}-s_{r-2}$ times,

$\left(m \sigma_{r}\right)$ occurring $s_{r}-s_{r-1}$ times.

- Let $\tau_{i}=\sigma_{r}+\sigma_{r-1}+\ldots+\sigma_{i}, 1 \leq i \leq r$. Then from the refined flag we see that $\operatorname{dimim} N=n-m \tau_{1}$, and so $\operatorname{dim} \operatorname{ker} N=m \tau_{1}$.

As an associative $\mathbb{F}$-algebra $R=Z_{L}(T)$ has its nil-radical nil $R$, and $R / n i l R$ is a semi-simple $\mathbb{F}$-algebra. The elements of $R$ which map $\mathbb{V}_{i, j+1}$ into $\mathbb{V}_{i, j}$ clearly form a nilpotent ideal $I$ of $\mathrm{R}$. On the other hand, $R / I$ is clearly isomorphic to a direct product of $L\left(\mathbb{W}_{s_{i}}\right), i=1,2, \ldots r$. So $I$ is nil $R$. This is worth recording as a theorem.

THEOREM 3.3. Let $T$ be in $L(\mathbb{V})$, with $m_{T}(x)=p(x)^{d}$ where $p(x)$ is a monic irreducible polynomial in $\mathbb{F}[x]$. Let $\mathbb{E}=\mathbb{F}[x] /(p(x))$. Then $R=Z_{L}(T)$ considered as an associative algebra has its maximal semisimple quotient isomorphic to a direct sum of matrix rings $M_{\sigma_{i}}(\mathbb{E})$, where $\sigma_{i}$ are as defined above.

In particular,

- $\operatorname{dim} R / n i l R=m\left(\sigma_{1}^{2}+\sigma_{2}^{2}+\ldots \sigma_{r}^{2}\right)$.

4. Strongly Commuting Operators. Let $T$ be in $L(\mathbb{V})$. We say that an operator $S$ in $L(\mathbb{V})$ strongly commutes with $T$ if $S$ commutes with $T$, and leaves invariant every $T$-invariant subspace of $\mathbb{V}$. It is interesting to compare the following theorem with Frobenius' bicommutant theorem. It will be useful later on.

TheOREM 4.1. Let $T$ be in $L(\mathbb{V})$. An operator $S$ in $Z_{L}(T)$ strongly commutes with $T$ iff $S$ is in $\mathbb{F}[T]$.

Proof. The "if" part is clear. Conversely, suppose that $S$ in $Z_{L}(T)$ strongly commutes with $T$.

First consider the case when $(\mathbb{V}, T)$ is dynamically equivalent to $\left(\mathbb{F}[x] /\left(p(x)^{d}\right), \mu_{x}\right)$, where $p(x)$ is a monic irreducible polynomial in $\mathbb{F}[x]$. Let $S$ be in $Z_{L}(T)$, and $S(1)=$ $[f(x)]$. It is easy to see that $S=f(T)$. Thus $Z_{L}(T)=\mathbb{F}[T]$, and so every element in $Z_{L}(T)$ strongly commutes with $T$.

Next consider the case where $m_{T}(x)=p(x)^{d}$, and $p(x)$ is a monic irreducible polynomial in $\mathbb{F}[x]$. Then $\mathbb{V}$ is a direct sum of $T$-invariant subspaces $\mathbb{W}_{i}$, dynamically equivalent to $\left(\mathbb{F}\left[x_{i}\right] /\left(p\left(x_{i}\right)^{d_{i}}\right), \mu_{x_{i}}\right), 1 \leq i \leq k$, and $d=d_{1} \geq d_{2} \geq \ldots \geq d_{k}$. Let $e_{i}, 1 \leq i \leq k$ be a $T$-module generator in $\mathbb{W}_{i}$.

Let $\left.S\right|_{\mathbb{W}_{1}}=q_{1}(T)$ where $q_{1}(x)$ is a unique polynomial of degree at most $d m, m=$ $\operatorname{deg} p(x)$. For $j \geq 2$ let $q_{j}(x)$ be the polynomial of degree at most $d_{j} m$, such that $\left.S\right|_{\mathbb{W}_{j}}=q_{j}(T) e_{j}$. Then $S\left(e_{1}+e_{2}\right)=q_{1}(T) e_{1}+q_{j}(T) e_{j}$. On the other hand, since $S$ strongly commutes with $T$, we also have $S\left(e_{1}+e_{j}\right)=u(T)\left(e_{1}+e_{j}\right)$ for some polynomial $u(x)$ of degree at most $d m$. It follows that $\left(q_{1}(T)-u(T)\right) e_{1}=-\left(q_{j}(T)-u(T)\right) e_{j}$. 
Since $\mathbb{W}_{1} \cap \mathbb{W}_{j}=0$ we must have $\left(q_{1}(T)-u(T)\right) \equiv\left(q_{j}(T)-u(T)\right) \equiv 0\left(\bmod p(x)^{d_{j}}.\right)$ So $q_{1}(T) \equiv q_{j}(T)\left(\bmod p(x)^{d_{j}}\right.$.)

Finally consider the general case. Write $m_{T}(x)=\Pi_{i=1}^{r} p_{i}(x)^{d_{i}}$, where $p_{i}(x)$ 's are monic irreducible polynomials in $\mathbb{F}[x]$. Let $\mathbb{V}=\oplus \mathbb{V}_{i}$, where $\mathbb{V}_{i}=\operatorname{ker} p_{i}(x)^{d_{i}}$ be the corresponding primary decomposition of $\mathbb{V}$. Now $S$ leaves each $\mathbb{V}_{i}$ invariant. We have shown $\left.S\right|_{\mathbb{V}_{i}}=q_{i}(T)$ where $q_{i}(x)$ is a uniquely determined polynomial $\bmod p_{i}(x)^{d_{i}}$. By Chinese Remainder Theorem, there exists a uniquely determined polynomial $q(x)$ $\bmod m_{T}(x)$ which is congruent to $q_{i}(x) \bmod p_{i}(x)^{d_{i}}$. This completes the proof.

5. Lifting $T$-invariant $\mathbb{E}$-structures, and "S+N"-decomposition. Let $T$ be in $L(\mathbb{V})$, and $\mathbb{E}$ an extension field of $\mathbb{F}$. An $\mathbb{E}$-structure on $\mathbb{V}$ is an $\mathbb{F}$-algebra homomorphism $\sigma_{\mathbb{E}}: \mathbb{E} \rightarrow L(\mathbb{V})$. Such a homomorphism is necessarily injective, and allows one to consider $\mathbb{V}$ as a vector space over $\mathbb{E}$, lifting the structure of $\mathbb{V}$ as a vector space over $\mathbb{F}$. An $\mathbb{E}$-structure $\sigma_{\mathbb{E}}$ is said to be $T$-invariant if the image of $\sigma_{\mathbb{E}}$ lies in $Z_{L}(T)$.

Suppose that $m_{T}(x)=p(x)^{d}$, where $p(x)$ is a monic irreducible polynomial in $\mathbb{F}[x]$. Let $\mathbb{E}=\mathbb{F}[x] /(p(x))$. An interesting problem is to investigate when $\mathbb{V}$ admits a $T$ invariant $\mathbb{E}$-structure. When $d=1, T$ itself induces a canonical $\mathbb{E}$-structure. Namely, $\mathbb{F}[T] \approx \mathbb{E}$, and the inclusion mapping of $\mathbb{F}[T]$ in $Z_{L}[T]$ is a $T$-invariant $\mathbb{E}$-structure. Assume $d \geq 2$. Then $\mathbb{V}_{i} / \mathbb{V}_{i-1}$ admits a canonical $T$-invariant $\mathbb{E}$-structure, since the minimal polynomial of the operator induced by $T$ on $\mathbb{V}_{i} / \mathbb{V}_{i-1}$ is $p(x)$. Our concern is whether these canonical $\mathbb{E}$-structures on $\mathbb{V}_{i} / \mathbb{V}_{i-1}$ 's can be lifted to a canonical $\mathbb{E}$-structure on $\mathbb{V}$ itself. By a "canonical $\mathbb{E}$-structure on $\mathbb{V}$ " we mean:

i) Each $T$-invariant subspace is an $\mathbb{E}$-subspace.

ii) For each $i=1,2, \ldots, d$, the induced $\mathbb{E}$-structure on $\mathbb{V}_{i} / \mathbb{V}_{i-1}$ coincides with the one induced by $T$.

It will eventually turn out that if $\mathbb{V}$ admits an $\mathbb{E}$-structure which satisfies ii) then it also satisfies i). A first basic result in this direction is the following. For its importance in the theory of algebraic groups see below. In the following, for $f(x)$ in $\mathbb{F}[x]$, let $f^{\prime}(x)$ denote its formal derivative.

THEOREM 5.1. Let $T$ be in $L(\mathbb{V}), m_{T}(x)=p(x)^{d}$, where $p(x)$ is a monic irreducible polynomial in $\mathbb{F}[x]$, and $\mathbb{E}=\mathbb{F}[x] /(p(x))$. Then $\mathbb{V}$ admits a T-invariant $\mathbb{E}$-structure iff either $d=1$ or $p^{\prime}(x)$ is not identically zero. Such structure is unique if it is canonical in the sense that it satisfies i) and ii) stated above.

Proof. First we consider the issue of the existence of a $T$-invariant $\mathbb{E}$-structure. We may assume $d \geq 2$. If $\operatorname{deg} p(x)=1$, we have $\mathbb{E}=\mathbb{F}$, and $p(x)=x-\alpha$ for some $\alpha$ in $\mathbb{F}$. On each $\mathbb{V}_{i+1} / \mathbb{V}_{i}, T$ acts as $\mu_{\alpha}: v \mapsto \alpha v$. Then clearly $\tilde{\mu}_{\alpha}$ defined by the same formula acting on $\mathbb{V}$ is a $T$-invariant $\mathbb{E}$-structure on $\mathbb{V}$. Note that we have also $p^{\prime}(x) \equiv 1 \neq 0$, and the structure is canonical. So suppose $\operatorname{deg} p(x)=m \geq 2$.

First suppose that $p^{\prime}(x)$ is not identically 0 . Then $\operatorname{deg} p^{\prime}(x) \leq m-1$. So $p(x), p^{\prime}(x)$ are relatively prime.

Since $(\mathbb{V}, T)$ is dynamically equivalent to a direct sum of pairs of the form $\left(\mathbb{F}[x] /\left(p(x)^{e}, \mu_{x}\right)\right.$ where $e \leq d$, and $\mu_{x}([u(x)]) \mapsto([x u(x)])$, it suffices to prove the existence of $\mu_{x}$-invariant $\mathbb{E}$-structure in the special case of $\left(\mathbb{F}[x] /\left(p(x)^{e}\right), \mu_{x}\right), e \geq 2$. In this case $Z_{L}\left(\mu_{x}\right) \approx \mathbb{F}[x] /\left(p(x)^{e}\right)$. For any $y \in \mathbb{F}[x]$ let $[y]$ denote its class in $\mathbb{F}[x] /\left(p(x)^{e}\right)$. So the assertion of existence of an $\mu_{x}$-invariant $\mathbb{E}$-structure amounts to the existence of a polynomial $z=u(x) \in \mathbb{F}[x]$ such that the corresponding operator $\mu_{z}$ has minimal polynomial $p(x)$. 
Since $p(x), p^{\prime}(x)$ are relatively prime, there exist $a(x), b(x) \in \mathbb{F}[x]$ such that $a(x) p(x)+b(x) p^{\prime}(x)=1$. Consider $y=x-b(x) p(x)$. (Notice that for any polynomial $u(x)$ in $\mathbb{F}[x], \mu_{u(x) p(x)}$ is nilpotent, and its minimal polynomial is of the form $x^{r}, r \leq d$.) Writing $\epsilon=-b(x) p(x)$, the formal Taylor's theorem (for polynomials with coefficients in commutative rings), gives

$$
\begin{gathered}
p(y)=p(x+\epsilon)=p(x)+\epsilon p^{\prime}(x)+\frac{\epsilon^{2}}{2} p^{\prime \prime}(x)+\ldots \\
\equiv p(x)\left(1-b(x) p^{\prime}(x)\right)+\ldots \equiv p(x)(a(x) p(x))+\ldots \equiv 0\left(\bmod p(x)^{2}\right) .
\end{gathered}
$$

So $p(y)^{r}=0$, for a suitable $r<e$. It follows that $\mu_{y}$ has minimal polynomial of the form $p(x)^{r}$ where $r<e$. So $\mathbb{F}[[y]] \approx \mathbb{F}[x] /\left(p(x)^{r}\right)$, and $\mathbb{F}[[y]] \subset \mathbb{F}[[x]]$. By induction on $e$ it follows that there exists a polynomial $z=u(x) \in \mathbb{F}[x]$ such that the corresponding operator $\mu_{z}$ has minimal polynomial $p(x)$.

To prove the converse suppose that we have a pair $(\mathbb{V}, T), m_{T}(x)=p(x)^{d}, d \geq 2$ where $p(x)$ is a monic irreducible polynomial in $\mathbb{F}[x], \mathbb{E}=\mathbb{F}[x] /(p(x))$, and $\mathbb{V}$ admits a $T$-invariant $\mathbb{E}$-structure. This implies the existence of $S$ in $Z_{L}(T)$ with $m_{S}(x)=p(x)$. In the associated flag $\mathbb{V}_{2}$ is $S$-invariant. We only need to prove that $p^{\prime}(x) \not \equiv 0$. So we readily reduce to the case $d=2$. To arrive at a contradiction, suppose that $p^{\prime}(x) \equiv 0$. Now notice that for any polynomial $u(x)$ in $\mathbb{F}[x]$ we have by the formal Taylor's theorem,

$$
p(S+u(T))=p(S)+u(T) p^{\prime}(S)+\ldots=p(S)=0 .
$$

But then

$$
p(T)=p(S+T-S)=p(S+T)-S p^{\prime}(S+T)+\ldots=p(S+T)=0 .
$$

This is a contradiction since we have assumed $m_{T}(x)=p(x)^{2}$. So we must have $p^{\prime}(x) \not \equiv 0$.

Next we consider the issue of uniqueness of a canonical $T$-invariant $\mathbb{E}$-structure. Let $\sigma_{1}: \mathbb{E} \rightarrow Z_{L}(T), \sigma_{2}: \mathbb{E} \rightarrow Z_{L}(T)$, be two canonical $T$-invariant $\mathbb{E}$-structures. By passing to a $T$-invariant subspace, we may reduce to the case when $(\mathbb{V}, T)$ is dynamically equivalent to $\left.\mathbb{F}[x] /\left(p(x)^{d}\right), \mu_{x}\right)$. Then $Z_{L}(T)=\mathbb{F}(T)$. Let $\alpha$ be a primitive element of $\mathbb{E}$ over $\mathbb{F}$, and $\sigma_{i}(\alpha)=S_{i}, i=1,2$. Let $S_{i}=f_{i}(T)$ where $f_{i}(x) \in \mathbb{F}[x]$ are well-defined polynomials mod $p(x)^{d}$. Since $S_{i}$ 's define canonical $T$-invariant $\mathbb{E}$ structures we see that we must have $f_{i}(x)=x \bmod p(x)$. In the existence proof we considered polynomials $a(x), b(x)$ satisfying $a(x) p(x)+b(x) p^{\prime}(x)=1$. Notice that $b(x)$ is uniquely defined by the condition that $\operatorname{deg} b(x)<m$. This is also the unique choice so that $p(x-b(x) p(x)) \equiv 0 \bmod p(x)^{2}$. The same argument shows that the induction procedure used in the existence proof leads to a polynomial $f(x)$ uniquely determined mod $p(x)^{d}$, such that $m_{f(T)}(x)=p(x)$. So $f(x)=f_{1}(x)=f_{2}(x)$, and hence $\sigma_{1}=\sigma_{2}$. This finishes the proof.

REMARK 5.2. Notice that the condition $p^{\prime}(x) \not \equiv 0$ is automatically satisfied if the characteristic of $\mathbb{F}$ is 0 . Suppose that the characteristic of $\mathbb{F}$ is $l>0$. Let $p(x)=$ 
$\sum_{i=0}^{m} a_{i} x^{i}$, Then $p^{\prime}(x) \equiv 0$ iff $a_{i}=0$ unless $i$ is a multiple of $l$. So if $p^{\prime}(x) \equiv 0$, then we may take $p(x)=\sum_{i=0}^{m^{\prime}} b_{i} x^{i l}$, where $m=m^{\prime} l$. When $l>0, \mathbb{F}$ is said to be perfect if $u \mapsto u^{l}$ is an isomorphism. For example a finite field, or an algebraically closed filed is automatically perfect. Notice that the condition $p^{\prime}(x) \not \equiv 0$ is automatically satisfied if $\mathbb{F}$ is perfect. For otherwise, we can write $b_{i}=c_{i}^{l}$ and so $p(x)=\left(\sum_{i=0}^{m^{\prime}} c_{i} x^{i}\right)^{l}$, which will contradict that $p(x)$ is irreducible over $\mathbb{F}$.

Definition 5.3. Let $T$ be in $L(\mathbb{V})$. A " $\mathrm{S}+\mathrm{N}$ "-decomposition of $T$ is a pair $S, N$ such that i) $T=S+N$, ii) $S$ is dynamically semi-simple, iii) $N$ is nilpotent, and iv) $S N=N S$.

REMARK 5.4. Usually this notion is defined where dynamic semisimplicity is replaced by a stronger condition of algebraic semisimplicity, cf. the remarks in the introduction. This notion is basic in the theory of algebraic groups, cf. [1], [4], cf. also [2] for historical remarks.

TheOREM 5.5. Let $T$ be in $L(\mathbb{V})$, and $m_{T}(x)=\Pi_{i=1}^{r} p_{i}(x)^{d_{i}}$, where $p_{i}(x)$ 's are monic irreducible polynomials in $\mathbb{F}[x]$. Then

1) $T$ admits a " $S+N$ "-decomposition iff for each $i$, either $d_{i}=1$ or else $p_{i}^{\prime}(x) \not \equiv 0$.

2) If it exists, a "S+N"-decomposition is unique.

3) If $m_{T}(x)=p(x)^{d}, p(x)$ is a monic irreducible polynomial, $\mathbb{E}=\mathbb{F}[x] /(p(x))$, and $a$ " $S+N$ "-decomposition exists, then $S$ defines the canonical $T$-invariant $\mathbb{E}$-structure on $\mathbb{V}$. In particular $S$ strongly commutes with $T$, and so $S$, and hence $N$, are polynomials in $T$.

Proof. Notice that by the condition iv) in the definition of "S+N"-decomposition, $S, N$ are in $Z_{L}(T)$. So they leave the $T$-primary decomposition of $\mathbb{V}$ invariant, and their restriction to a $T$-primary component of $\mathbb{V}$ are the semisimple and nilpotent components of the restriction of $T$. So to investigate the existence of " $\mathrm{S}+\mathrm{N}$ "-decomposition we reduce to the case where $m_{T}(x)=p(x)^{d}$, where $p(x)$ is a monic irreducible polynomial in $\mathbb{F}[x]$.

First consider the existence issue. If $d=1$, then $T$ is semisimple, and taking $S=T$, and $N=0$, we obtain a "S+N"-decomposition. So consider $d \geq 2$. Let $\mathbb{E}=\mathbb{F}[x] /(p(x))$. First suppose that $p^{\prime}(x) \not \equiv 0$. In the previous theorem we observed that under this condition there exists a polynomial $f(x)$ in $\mathbb{F}[x]$ such that $S=f(T)$ defines a canonical $T$-invariant $\mathbb{E}$-structure on $\mathbb{V}$. In particular $m_{S}(x)=p(x)$, and so $S$ is dynamically semisimple. Let $\bar{T}_{i}, \bar{S}_{i}$, be the operators induced by $T, S$ respectively on $\mathbb{V}_{i}=\operatorname{ker} p(T)^{i}, i=0,1,2, \ldots, d$. Since $S$ defines a canonical $T$-invariant $\mathbb{E}$-structure we have $\bar{T}_{i}=\bar{S}_{i}$. It follows that $N=T-S$ is nilpotent, and hence $T=S+N$ is an "S+N"-decomposition of $T$.

Conversely suppose $T=S+N$ is an "S+N"-decomposition of $T$. Then the induced operatots $\bar{T}_{i}, \bar{S}_{i}$, on $\mathbb{V}_{i}, i=1,2, \ldots, d$, are commuting dynamically semisimple operators. So their nilpotent difference $\bar{N}_{i}$ must be 0 . (See equation (5.1) below). So $m_{\bar{T}_{i}}(x)=p(x)=m_{\bar{S}_{i}}(x)$. Since $S$ is dynamically semi-simple, it follows that $m_{S}(x)=p(x)$ also. Let $\mathbb{E}=\mathbb{F}[x] /(p(x))$. Thus $\mathbb{F}[S] \approx \mathbb{E}$, and $S$ defines a $T$-invariant $\mathbb{E}$-structure on $\mathbb{V}$. So $p_{i}^{\prime}(x) \not \equiv 0$.

Now consider the issue of uniqueness of "S+N"-decomposition. Again we reduce to the case when $m_{T}(x)=p(x)^{d}$. Let $d=1$. Then $S=T, N=0$ is one " $\mathrm{S}+\mathrm{N}$ "decomposition. Suppose $T=S+N$ any "S+N"-decomposition. We need to show 
that $N=0$. Indeed,

$$
p(T)=p(S+N)=p(S)+N p^{\prime}(S)+\frac{N^{2}}{2 !} p^{\prime \prime}(S)+\ldots
$$

Notice that $p(T)=p(S)=0$, and $p^{\prime}(S)$ is invertible. If $N \neq 0$ then the rank of $N$ is greater than the rank of $N^{i}$ for $i \geq 2$. So the above equation is not possible unless $N=0$. Now consider the case $d \geq 2$. The proof is by induction on $d$. Let $T=S+N, T=S_{1}+N_{1}$ be two "S+N"-decomposition. By induction we may assume $S=S_{1}$ on $\mathbb{V}_{d-1}$, and $S, S_{1}$ induce the same operators on $\mathbb{V}_{d} / \mathbb{V}_{d-1}$. It follows that we must have $S_{1}=S+M$ where $M$ maps $\mathbb{V}_{d}$ into $\mathbb{V}_{d-1}$, and $V_{d-1}$ onto 0 . Such $M$ must be nilpotent.

$$
p\left(S_{1}\right)=p(S+M)=p(S)+M p^{\prime}(S)+\frac{M^{2}}{2 !} p^{\prime \prime}(S)+\ldots
$$

By the same argument as above we see that $M=0$. It follows that " $\mathrm{S}+\mathrm{N}$ "decomposition, if it exists, is unique.

By uniqueness of " $\mathrm{S}+\mathrm{N}$ "-decomposition it follows that when $m_{T}(x)=p(x)^{d}$, and "S+N"-decomposition exists, then $S$ defines the canonical $T$-invariant $\mathbb{E}$-structure. So $S$ strongly commutes with $T$, and hence it (and so also $N$ ) is a polynomial in $T$.

$\square$

REMARK 5.6. The theorem 5.4 shows that in the definition of canonical $T$ invariant $\mathbb{E}$-structure the condition i) is a consequence of condition ii). On the other hand, to get uniqueness of a $T$-invariant $\mathbb{E}$-structure it is clearly necessary to impose a condition such as ii). For example, if $m_{T}(x)$ and $m_{S}(x)$ are both irreducible, such that $\mathbb{E}=\mathbb{F}[x] /\left(m_{T}(x)\right) \approx \mathbb{F}[x] /\left(m_{S}(x)\right)$ then $T$ and $S$ would usually define different $\mathbb{E}$-structures.

REMARK 5.7. As remarked earlier, perfectness of $\mathbb{F}$ is a sufficient condition for the existence of " $\mathrm{S}+\mathrm{N}$ "-decomposition. However the author is not aware of a statement of a necessary and sufficient condition for the existence of " $\mathrm{S}+\mathrm{N}$ "-decomposition in the literature. One may avoid the issue by defining a different notion of semisimplicity, namely "algebraic semi-simplicity" to mean that the operator is diagonalizable over the algebraic closure of $\mathbb{F}$. However, in author's opinion, it is desirable to have all elements of the orthogonal group with respect to an anisotropic quadratic form to be "semisimple". This would not be the case if we take the algebraic notion of semisimplicity. We also note that among the fields of positive characteristic, an important class of fields, namely, function fields of algebraic varieties of positive dimensions over finite fields, are not perfect. Lastly we note that there are misleading remarks in the literature that the dynamic and algebraic notions of semisimplicity are equivalent.

REMARK 5.8. Finally we would like to remark on a forgotten rational canonical form for matrices due to Wedderburn, [11]. In the standard texts on Algebra, such as [8], the authors present a matrix for an operator by choosing a suitable basis, called its rational canonical form. Let $T$ be in $L(\mathbb{V})$, and $m_{T}(x)=p(x)^{d}$, where $p(x)$ is a monic irreducible polynomial in $\mathbb{F}[x]$. For some authors the matrix presented to represent $T$ involves a companion matrix of $p(x)^{d}$. This is obviously a poor choice when $d \geq 2$. It is better to use only the companion matrix of $p(x)$. It is worth noting that one may use any matrix conjugate to a companion matrix. (This remark is important even in 
the basic case when $\mathbb{F}=\mathbb{R}$, the field of real numbers, and important for the solutions of linear first order ODEs with constant coefficients, cf. section 6.) But secondly when $\operatorname{deg} p(x)=m \geq 2$, and $d \geq 2$, the matrix presented is written in the form "S+N" where $S$ (the matrix of diagonal blocks) is semisimple and $N$ (the matrix of off-diagonal blocks) is nilpotnt. However this is not the "S+N"-decomposition of $T$, for these $S, N$ do not commute. In case "S+N"-decomposition exists, a better matrix representation is obtained by choosing the off-diagonal blocks to be identity matrices of size $m \times m$. Such a basis may be constructed starting from an $\mathbb{E}$-basis which gives the usual "Jordan block" over $\mathbb{E}$, and then constructing the corresponding basis over $\mathbb{F}$. This was effectively mentioned already by Wedderburn, cf. [11], and rediscovered by the author early on in this investigation. The author thanks Rony Gouraige for pointing out the reference [11].

6. The Affine Case. In this section we extend the theory to the affine case, and determine the centralizer of an affine map.

Let $\mathbb{F}$ and $\mathbb{V}$ be as in the introduction, $\mathbb{A}$ the underlying affine case, and $T=(A, v)$ an affine map which maps $x$ to $A x+v$. As observed there, $A$ in $(A, v)$ has an intrinsic affine meaning, and $v$ has an intrinsic affine meaning if $A=I$. Let $S=(\alpha, a), \alpha \in$ $G L(\mathbb{V})$ be an element of $G A(\mathbb{V})$. Then

$$
S^{-1}=\left(\alpha^{-1},-\alpha^{-1} a\right),
$$

and

$$
S T S^{-1}=\left(\alpha A \alpha^{-1},-\alpha A \alpha^{-1} a+\alpha v+a\right) .
$$

Let $\mathcal{C}_{L}(\mathbb{V})$ resp. $\mathcal{C}_{A}(\mathbb{V})$ denote the orbit-spaces $L(\mathbb{V}) / G L(\mathbb{V})$ resp. $A(\mathbb{V}) / G A(\mathbb{V})$. For $T$ in $L(\mathbb{V})$ resp. $A(\mathbb{V})$ let $[T]_{L}$ resp. $[T]_{A}$ denote its orbit in $\mathcal{C}_{L}(\mathbb{V})$ resp. $\mathcal{C}_{A}(\mathbb{V})$. We have seen that the map $(A, v) \mapsto A$ is a homomorphism $l: A(\mathbb{V}) \rightarrow L(\mathbb{V})$. The formula (6.2) shows that the map $[(A, v)]_{A} \mapsto[A]_{L}$ is a well-defined map $[l]: \mathcal{C}_{A}(\mathbb{V}) \rightarrow \mathcal{C}_{L}(\mathbb{V})$. The main result about the map $[l]$ is

THEOREM 6.1. [l] is a finite map, that is $[l]^{-1}([A])$ has only finitely many elements. More precisely, for $A \in L(\mathbb{V})$ let $m_{A}(x)=(x-1)^{r} g(x)$, where $g(1) \neq 0$ be its minimal polynomial. Here $r \geq 0$ is an integer. Then $[l]^{-1}([A])$ has $r+1$ elements.

Proof. First consider the generic case where $r=0$. Consider the equation $(*) A x+$ $v=x$ where $x$ is indeterminate, and $A \in L(\mathbb{V}), v \in \mathbb{V}$ are known entities. Since $r=0$, we have $\operatorname{det}(I-A) \neq 0$. So $\left(^{*}\right)$ has a unique solution in $x$. Let $x_{0}$ be that unique solution. Let $\tau=\left(I, x_{0}\right)$. Then $\tau(A, v) \tau^{-1}=(A, 0)=A$. So any element in $l^{-1}(A)$ is conjugate to $A$. It follows that $[l]^{-1}([A])$ has a unique element.

Now suppose $r>0$. Then $\mathbb{V}=\mathbb{V}_{1}+\mathbb{V}_{2}$ (direct sum) where $\mathbb{V}_{1}=\operatorname{ker}(A-I)^{r}$, and $\mathbb{V}_{2}=\operatorname{ker} g(A)$. Consider $T=(A, v)$. Write $v=v_{1}+v_{2}$ where $v_{i} \in \mathbb{V}_{i}, i=1,2$. Let $x_{0}$ be the solution in $\mathbb{V}_{2}$ of the equation $(*) A x+v_{2}=x$. Such solution exists since $\left.\operatorname{det}(I-A)\right|_{\mathrm{V}_{2}} \neq 0$. Let $\tau=\left(I, x_{0}\right)$. Then $\tau(A, v) \tau^{-1}=\left(A, v_{1}\right)$. We have proved that an element $(A, v) \in l^{-1}(A)$ is in the same $G A(\mathbb{V})$-orbit as an element $\left(A, v_{1}\right)$, where $(A-I)^{r}\left(v_{1}\right)=0$. Let $s$ be the least non-negative integer, $s \leq r$, such that $(A-I)^{s}\left(v_{1}\right)=0$. Now the theorem follows from the following lemma. 
Lemma 6.2. Suppose $S=(A, v)$ resp. $T=(A, w)$ be in $A(\mathbb{V})$ such that $m_{A}(x)=$ $(x-1)^{r}$. Let $s$ resp. $t$ be the least non-negative integers $\leq r$ satisfying $(A-I)^{s}(v)=0$ resp. $(A-I)^{t}(w)=0$. Then $S$ and $T$ are in the same $G A(\mathbb{V})$-orbit iff $s=t$.

Proof. From (6.2) we see that $(\alpha, a)$ conjugates $S$ into $T$ iff $\alpha$ is in $Z_{L}(A)^{*}$ and $w=(I-A) a+\alpha v$. Since $m_{A}(x)=(x-1)^{r}$ we are in the situation of the previous section. In particular, set $N=I-A$, and consider the $Z_{L}(A)$-invariant refined flag. By symmetry, we may assume $s \leq t$. In the notation introduced in the previous section, let $\mathbb{V}_{t}=\mathbb{V}_{t-1, k}$, and $v$ lies in $\mathbb{V}_{t}-\mathbb{V}_{t-1, k-1}$. From the structure of invertible elements in $Z_{L}(A)$, we see that $\alpha$ is in $Z_{L}(A)^{*}$ and $w=(I-A) a+\alpha v$ iff $s=t$.

Now we are in a position to determine the centralizer of an affine map. In effect, we describe a good representative of a $G A(\mathbb{V})$-orbit of the centralizer of an affine map.

Let $T=(A, v)$ in $A(\mathbb{V})$. Let $S=(B, w)$ be in $Z_{A}(T)$. The equation $S T=T S$ is equivalent to

i) $B A=A B$, i.e. $B \in Z_{L}(T)$.

ii) $B v+w=A w+v$, or $(B-I) v=(A-I) w$.

Case 1) Assume that $T$ has a fixed point. Then by conjugation by an element in $G A(\mathbb{V})$ (or what amounts to the same, by an affine change of co-ordinates) we may take $v=0$. With this choice, we take the flag associated to $A$. From ii) we see that

$$
Z_{A}(T)=\left\{(B, w) \mid B \in Z_{L}(A), \text { and } w \in \mathbb{V}_{1}\right\}
$$

where $\mathbb{V}_{1}=\operatorname{ker}(A-I)$.

Case 2) Assume that $T$ has no fixed point. Then again by change of affine coordinates by theorem (6.1) we may assume that $m_{A}(x)=(x-1)^{r} g(x), g(1) \neq 0$ is the minimal polynomial of $A$, and $s$ is the least positive integer such that $(A-I)^{s} v=0$. The equation ii) implies that

$$
(A-I)^{s}(B-I) v=(B-I)(A-I)^{s} v=0=(A-I)^{s+1} w .
$$

So $w$ is in $\mathbb{V}_{s+1}$, where $\mathbb{V}_{i}=\operatorname{ker}(A-I)^{i}$.

Conversely, suppose that $w$ is in $\mathbb{V}_{s+1}$. Then we show that there exists a $B$ in $Z_{L}(A)$ such that $(B, w)$ is in $Z_{A}(T)$, and we can precisely determine $B$ 's having this property. Indeed, in the double-subscript notation of the flag, $\mathbb{V}_{s}=\mathbb{V}_{s-1, k}$ (for a suitable $k), v$ is in $\mathbb{V}_{s}-\mathbb{V}_{s-1, k-1}$, and $(A-I) w$ is in $\mathbb{V}_{s}$. So there exists $C$ in $Z_{L}(T)$ so that $C v=(A-I) w$, and all such $C$ 's can be determined from the refined flag. For each such choice of $C$, we can then take $B=C+I$. These are precisely the $(B, w)$ 's in $Z_{A}(T)$.

Notice moreover that $(B, w)$ is in $Z_{A}(T)^{*}$ iff $B$ is in $Z_{L}(A)^{*}$. Assume that this is the case, then $B v$ is in $\mathbb{V}_{s}-\mathbb{V}_{s-1, k-1}$. Now equation ii) $(B-I) v=(A-I) w$ shows that $B v=v+(A-I) w$. Since $(A-I) w$ is in $\mathbb{V}_{s-1, k-1}$ we see that $B v \equiv v$ $\bmod \mathbb{V}_{s-1, k-1}$. It follows that the linear map $\bar{B}$ induced by $B$ on $\mathbb{V}_{s} / \mathbb{V}_{s-1, k-1}$ has eigenvalue 1 . So $B$ also has eigenvalue 1 , and the $N$-images of the corresponding eigen-vector show that the multiplicity of the eigenvalue 1 is at least $s$.

Summarizing, we have proved the following result.

Theorem 6.3. Let $T=(A, v)$ be in $A(\mathbb{V})$. Let $\mathbb{V}_{i}=\operatorname{ker}(A-I)^{i}$. 
1) If $T$ has a fixed point then $Z_{A}(T)$ is conjugate to

$$
\left\{(B, w) \mid B \in Z_{L}(A), \text { and } w \in \mathbb{V}_{1}\right\}
$$

2) Suppose $T$ has no fixed point, and $m_{A}(x)=(x-1)^{r} g(x), g(1) \neq 0$ is the minimal polynomial of $A$. Let $s \leq r$ be the least positive integer such that $(A-I)^{s} v=$ 0. Then $Z_{A}(T)$ is conjugate to

$$
\left\{(B, w) \mid B \in Z_{L}(A), w \in \mathbb{V}_{s+1},(B-I) v=(A-I) w .\right\}
$$

An element $(B, w)$ in $Z_{A}(T)^{*}$ necessarily has eigenvalue 1 with multiplicity at least s.

Remark 6.4. Suppose that $T=(A, v)$ in $A(\mathbb{V})$ has no fixed point. Then the explicit forward orbit-structure of $T$, or the orbit structure of $Z_{A}(T)^{*}$, is quite complicated, compared to the neat answer we obtained in case $T$ has a fixed point. However, next to orbit-structure, for some intuitive understanding, we can enquire about the invariant sets. On this score we have some satisfactory information. Namely, if $(B, w)$ is in $Z_{A}(T)$ then $B$ preserves the refined flag determined by $A$ in $\mathbb{U}=$ $\operatorname{ker}(A-I)^{r}$, where $m_{A}(x)=(x-1)^{r} g(x), g(1) \neq 0$, is the minimal polynomial of $A$. The affine translates of each of the subspaces in the flag may be called a family of affine flags in $\mathbb{U}$. Clearly $(B, w)$ preserves this family of affine flags as a whole.

In case the integer $s$ associated to $v$ in $(A, v)$ is 1 , one can say a bit more. Namely consider the $Z_{A}(T)$-invariant family of affine subspaces parallel to $\mathbb{V}_{1}$. Among these subspaces, there is actually one $Z_{A}(T)$-invariant subspace. Namely, up to an affine change of co-ordinates we may assume that $v$ is actually an eigenvector of $A$. Then the eigen-space $\mathbb{V}_{1}=\operatorname{ker}(A-I)$ itself is $Z_{A}(T)$-invariant.

Remark 6.5. Consider the case $\mathbb{F}=\mathbb{R}$, the field of real numbers, or $\mathbb{F}=\mathbb{C}$ the field of complex numbers. On the Lie algebra level, one may ask for "normal forms" of the solutions of affine vector fields on $\mathbb{A}$. This amounts to solutions of the ODEs

$$
\frac{d x}{d t}=A x+v, A \in L(\mathbb{V}), v \in \mathbb{V} .
$$

Equivalently one may ask for normal forms of representatives of conjugacy classes of one-parameter subgroups of $G A(\mathbb{V})$. In the texts on ODEs, cf. for example [5], this ODE is solved by the method of variation of parameters. The ideas in this section provide a short-cut. Namely consider the affine map $(A, v)$. If this map has a fixed point, (which is the case if $\operatorname{det}(A-I) \neq 0$ ), then by an affine change of coordinates we can make $v=0$, and the solutions are orbits of the one parameter group $t \mapsto e^{t A}$ in the new coordinate system. If the map has no fixed point then $A$ must have eigenvalue 1 . Write $m_{A}(x)=(x-1)^{r} g(x), g(1) \neq 0$. Let $\mathbb{R}^{n}=\mathbb{V}=\mathbb{V}_{1}+\mathbb{V}_{2}$, where $\mathbb{V}_{1}=\operatorname{ker}(A-I)^{r}, \mathbb{V}_{2}=\operatorname{ker} g(A)$. Let $v=v_{1}+v_{2}$, where $v_{i}$ is in $\mathbb{V}_{i}$ for $i=1,2$. By an affine change of coordinates we can make $v_{2}=0$. Choose the least positive integer $s$ such that $(A-I)^{s} v_{1}=0$. Then in the new coordinate system, the solutions are orbits of the one-parameter group

$$
t \mapsto\left(e^{t A}, t v_{1}+\frac{t^{2}}{2 !} A v_{1}+\frac{t^{3}}{3 !} A^{2} v_{1}+\ldots+\frac{t^{s}}{s !} A^{s-1} v_{1}\right)
$$

The point is that one can always make the translational part of a one-parameter group of the affine group a polynomial, rather than an infinite series, in $t$, either by conjugacy 
in $G A(\mathbb{V})$, or what is the same, by an appropriate affine change of coordinates. This "normal form" of a one-parameter group indicates that its orbits, or the orbits of its centralizer, in $\mathbb{V}$ are more complicated than in the linear case, when there is an "unavoidable" translational part, which carries an affine meaning.

REMARK 6.6. From a computational, or algorithmic, perspective the decomposition $\mathbb{R}^{n}=\mathbb{V}=\mathbb{V}_{1}+\mathbb{V}_{2}$, is readily computable. The main issue is the computation of $e^{t A}$. Now $m_{A}(x)$ is algorithmically computable as the last non-zero diagonal entry in the Smith normal form of the characteristic matrix $x I-A$. Assume that we have a factorization of $m_{A}(x)$ into its irreducible factors. When $\mathbb{F}=\mathbb{R}$ the irreducible factors are of degree 1 or 2 . The (generalized) eigenspaces corresponding to linear factors and the corresponding refined lattice of $Z_{L}(A)$-invariant subspaces, the corresponding (Jordan) canonical forms and their exponentials are all algorithmically computatble. When $\mathbb{F}=\mathbb{R}$ and $m_{A}(x)$ has irreducible factors of degree 2 , again the corresponding refined lattice of $Z_{L}(A)$-invariant subspaces is algorithmically computable. However the suggested rational canonical form in the texts of algebra using the companion matrix of an irreducible factor is not useful for computation of the exponential. If the irreducible factor is $x^{2}-2 a x+b, a^{2}-b<0$, then its companion matrix is $\left[\begin{array}{cc}0 & -b \\ 1 & 2 a\end{array}\right]$. It is decisively better to use the matrix $\left[\begin{array}{cc}a & -c \\ c & a\end{array}\right], a^{2}+c^{2}=b$ which is conjugate to the companion matrix. For then its exponential becomes readily computable, namely, $e^{a}\left[\begin{array}{cc}\cos c & -\sin c \\ \sin c & \cos c\end{array}\right]$. Also one should use the (forgotten) rational form as explained in section 6 , where the non-diagonal blocks are $2 \times 2$ identity matrices. This is indicated in the texts and exercises in [5] and [7], without adequate explanation.

7. Parametrization Theorems. As stated in the introduction, we have interpreted the phrase "understanding the dynamics" in our set-up to mean the parametrizations of similarity classes, $z$-classes, and finally the elements in $L(\mathbb{V})$ and $A(\mathbb{V})$ themselves in terms of objects having significance independent of the choices of linear or affine co-ordinate systems. Here the word "parametrization" is used in the following sense. The sets $L(\mathbb{V})$ and $A(\mathbb{V})$ are the "unknown" sets which we wish to understand in terms of the "known" sets $\mathbb{F}$ and $\mathbb{V}$, and the "universally known" sets such as natural numbers, integers, rational numbers, and if one wishes, also real and complex numbers, and any other similar sets, and the sets derived from such sets by applying the allowable constructions in the model of "naive" set theory. Loosely speaking, the parameters having values in abelian groups are called "numerical parameters", and the others, such as decompositions into subspaces or flags, are called "spatial" parameters. In more abstract terms they are made precise in theorem 2.1 of [9].

The parametrizations that are obtained here are in terms of the "arithmetic" of $\mathbb{F}$ as reflected in the monic irreducible polynomials, and subspaces of $\mathbb{V}$. The datum of irreducible polynomial in $\mathbb{F}[x]$ of degree $m$ is equivalent to the datum of a simple field extension $\mathbb{E}$ of $\mathbb{F}$ such that $[\mathbb{E}: \mathbb{F}]=m$, and a primitive element $\alpha$ of $\mathbb{E}$ over $\mathbb{F}$. Starting with a monic irreducible polynomial $p(x) \in \mathbb{F}[x]$ we have $\mathbb{E}=\mathbb{F}[x] /(p(x))$, and $\alpha=[x]$, the class of $x$ in $\mathbb{F}[x] /(p(x))$. Conversely, given $(\mathbb{E}, \alpha)$, we get $p(x)$ as the minimal polynomial of $\mu_{\alpha}$ where $\mu_{\alpha}: \mathbb{E} \rightarrow \mathbb{E}, \mu_{\alpha}(u)=\alpha u$. Here $\mu_{\alpha}$ is regarded as a $\mathbb{F}$-linear map of the vector space $\mathbb{E}$ over $\mathbb{F}$. To be completely precise, to obtain a 
one-to-one correspondence between $p(x)$ and pairs $(\mathbb{E}, \alpha)$ we need to consider the $\mathbb{F}$ isomorphism classes of $(\mathbb{E}, \alpha)$ 's. Namely, the pairs $\left(\mathbb{E}_{1}, \alpha_{1}\right),\left(\mathbb{E}_{2}, \alpha_{2}\right)$, are $\mathbb{F}$-isomorphic if there exists an $\mathbb{F}$-isomorphism carrying $\alpha_{1}$ to $\alpha_{2}$. In particular if we fix $\mathbb{E}$ in its isomorphism class of field extensions of $\mathbb{F}$, then $\alpha$ is defined only up to the action of $G(\mathbb{E} / \mathbb{F})$, the group of $\mathbb{F}$-automorphisms of $\mathbb{E}$.

Let $n=\operatorname{dim} \mathbb{V}$ and $\pi: n=\sum_{i=1}^{r} n_{i}$ be a partition of $n$. A decomposition $\mathcal{D}_{\pi}$ patterned on the partition $\pi$ of $\mathbb{V}$ is a direct sum decomposition $\mathbb{V}=\oplus_{i=1}^{r} \mathbb{V}_{i}$ into subspaces, where $\operatorname{dim} \mathbb{V}_{i}=n_{i}$.

Let $n=\operatorname{dim} \mathbb{V}$. Let $m$ be a divisor of $n$, and $n=m l$. Let $r$ be a natural number, and $r$ pairs of natural numbers $\left\{\left(s_{1}, \sigma_{1}\right),\left(s_{2}, \sigma_{2}\right), \ldots\left(s_{r}, \sigma_{r}\right)\right\}$ such that $s_{1}<s_{2}<$ $\ldots<s_{r}$, and $l=\Sigma_{i=1}^{r} s_{i} \sigma_{i}$. A flag of type $\left(n, m ;\left\{\left(s_{1}, \sigma_{1}\right),\left(s_{2}, \sigma_{2}\right), \ldots\left(s_{r}, \sigma_{r}\right)\right\}\right)$ is an increasing family of subspaces $\mathbb{V}_{i, j}, 0 \leq i \leq r, 0 \leq j$ such that the successive quotients have dimensions: $\left(m \sigma_{r}, m \sigma_{r-1}, \ldots, m \sigma_{1}\right)$ occurring $s_{1}$ times, $\left(m \sigma_{r}, m \sigma_{r-1}, \ldots, m \sigma_{2}\right)$, occurring $s_{2}-s_{1}$ times, $\ldots\left(m \sigma_{r}, m \sigma_{r-1}\right)$ occurring $s_{r-1}-s_{r-2}$ times, $\left(m \sigma_{r}\right)$ occurring $s_{r}-s_{r-1}$ times.

Such a flag is denoted by $\mathcal{F}\left(\left(n, m ;\left\{\left(s_{1}, \sigma_{1}\right),\left(s_{2}, \sigma_{2}\right), \ldots\left(s_{r}, \sigma_{r}\right)\right\}\right)\right.$

Now suppose that there exists a simple field extension $\mathbb{E}$ of $\mathbb{F}$ such that $[\mathbb{E}: \mathbb{F}]=m$. Then since the dimension of each successive sub-quotient of $\mathcal{F}\left(n, m ;\left\{\left(s_{1}, \sigma_{1}\right),\left(s_{2}, \sigma_{2}\right), \ldots\left(s_{r}, \sigma_{r}\right)\right\}\right)$ is divisible by $m$, it has a structure of a vector space over $\mathbb{E}$. We denote such a choice of an $\mathbb{E}$-structure, a bit loosely, by $J_{\mathbb{E}}$. When

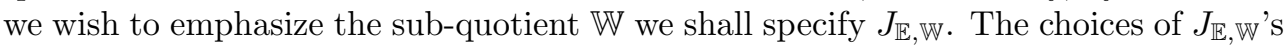
are by no means unique. In fact $G L(\mathbb{W})$ clearly acts on the set of $\mathbb{E}$-structures on $\mathbb{W}$. An important point, which is easy to see, is that the action of $G L(\mathbb{W})$ on the set of $\mathbb{E}$-structures is transitive.

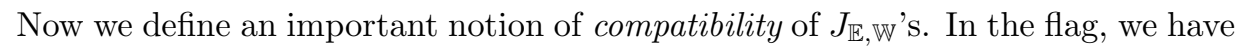

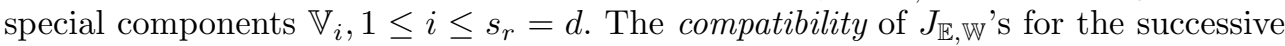
sub-quotients in the flag means that there are $\mathbb{E}$-structures on $\mathbb{V}_{i+1} / \mathbb{V}_{i}, 0 \leq i<d$ such that for all the components $\mathbb{V}_{i, j}$ in the chain from $\mathbb{V}_{i}$ to $\mathbb{V}_{i+1}$ the sub-quotients $\mathbb{V}_{i, j} / \mathbb{V}_{i}$ are $\mathbb{E}$-subspaces of $\mathbb{V}_{i+1} / \mathbb{V}_{i}$, and the $\mathbb{E}$-structure on $\mathbb{W}=\mathbb{V}_{i, j} / \mathbb{V}_{i, j-1}$ coincides with $J_{\mathbb{E}, \mathbb{W}}$. One may enquire whether the $\mathbb{E}$-structures on $\mathbb{V}_{i+1} / \mathbb{V}_{i}$, are similarly compatible with a single $\mathbb{E}$-structure on $\mathbb{V}$. As discussed in section 5 , this turns out to be a subtle point related to the existence of " $S+N$ "-decomposition.

With this preparation, we are in a position to describe our parametrizations.

ThEOREM 7.1. A) $A G L(\mathbb{V})$-orbit in its action on $L(\mathbb{V})$ is parametrized by the following data.

i) A primary partition $\pi: n=\sum_{i=1}^{r} n_{i}, n_{i}=m_{i} l_{i}$.

ii) The secondary partitions $l_{i}=\sum_{j=1}^{r_{i}} s_{i, j} \sigma_{i, j}$, where $s_{i, 1}<s_{i, 2}<\ldots s_{i, r_{i}}$.

iii) An $\mathbb{F}$-isomorphism class of pairs $\left(\mathbb{E}_{i}, \alpha_{i}\right)$, where $\mathbb{E}_{i}$ is a simple field extension of $\mathbb{F}$ of degree $m_{i}$ with $\alpha_{i}$ as its primitive element, for $i=1,2, \ldots, r$.

B) $A G A(\mathbb{V})$-orbit in its action on $A(\mathbb{V})$ is parametrized by the data i), ii), iii) as in $A$ ) and with $m(x)=(x-1)^{u} g(x), g(1) \neq 0$,

iv) A non-negative integer $s \leq u$.

THEOREM 7.2. : A) A z-class in the $G L(\mathbb{V})$-action on $L(\mathbb{V})$ is parametrized by the following data.

i) A primary partition $\pi: n=\sum_{i=1}^{r} n_{i}, n_{i}=m_{i} l_{i}$,

ii) The secondary partitions $l_{i}=\sum_{j=1}^{r_{i}} s_{i, j} \sigma_{i, j}$, where $s_{i, 1}<s_{i, 2}<\ldots s_{i, r_{i}}$. 
iii) Simple field extensions $\mathbb{E}_{i}, 1 \leq i \leq r$ of $\mathbb{F},\left[\mathbb{E}_{i}: \mathbb{F}_{i}\right]=m_{i}$.

B) $A$ z-class of $(A, v)$ in the $G A(\mathbb{V})$-action on $A(\mathbb{V})$ is parametrized by the data i), ii), iii) as in A) in case $m_{A}(x)$ does not have 1 as an eigenvalue. In case $m_{A}(x)=$ $(x-1)^{u} g(x), g(1) \neq 0$, and $u>0$, then the $z$-class of $(A, v)$ is parametrized by the data i), ii), iii) as in A) and

iv) A non-negative integer $s \leq u$.

TheOREM 7.3. A) An element of $L(\mathbb{V})$ is uniquely determined by the following data. The data i), ii), iii) of part A) in theorem 7.1, in particular the field extensions $\mathbb{E}_{i}=\mathbb{F}[x] /\left(p_{i}(x)\right)$, and the primitive elements $\alpha_{i}$.

iv) A decomposition $\mathcal{D}_{\pi}: \mathbb{V}=\oplus_{i=1}^{r} \mathbb{V}_{i}$ of $\mathbb{V}$ patterned on the primary partition $\pi$.

v) Flags $\mathcal{F}\left(\left(n_{i}, m_{i} ;\left\{\left(s_{i, 1}, \sigma_{i, 1}\right),\left(s_{i, 2}, \sigma_{i, 2}\right), \ldots\left(s_{i, r_{i}}, \sigma_{i, r_{i}}\right)\right\}\right)\right.$ of subspaces in $\mathbb{V}_{i}$, patterned on the secondary partitions.

vi) Compatible $\mathbb{E}_{i}$-structures on the sub-quotients in the flag in each $\mathbb{V}_{i}$.

$B)$ An element $T$ of $A(\mathbb{V})$ is uniquely determined by the following data.

Case 1. (T has a fixed point): Choose a fixed point as the origin. So T may be identified with an element in $L(\mathbb{V})$. The data i), ... , vi) in part $A$ ) is independent of the choice of the fixed point. These data and the affine subspace of fixed points determine $T$.

Case 2. ( $T$ has no fixed point): Express $T$ as $(B, v)$ so that there exists $s$ a least positive integer such that $(I-B)^{s} v=0$. Then the invariants i), ..., vi) in part $A$ ) associated to $B$ and $v$ uniquely determine $T$.

The proofs of theorems 7.1-7.3 are given in the next two sections. A major consequence of theorem 7.2 , cf. also section 10 , is the following theorem.

TheOREm 7.4. Let $\mathbb{V}$ be an $n$-dimensional vector space over a field $\mathbb{F}$. Suppose $\mathbb{F}$ has the property that there are only finitely many extensions of $\mathbb{F}$ of degree at most $n$. Then there are finitely many z-classes of $G L(\mathbb{V})$-, resp. $G A(\mathbb{V})$-, actions on $L(\mathbb{V})$, resp. $A(\mathbb{V})$.

8. Proof of Parametrization Theorems 7.1 and 7.3. We begin with the proof of Theorem 7.1. Notice that the data in ii), and iii) in part A) is just the numerical data regarding the exponents and multiplicities in the elementary divisors in the classical theory, which can be independently read from the refined flag. Given an element $T$ in $L(\mathbb{V})$, we associate to it

i) the minimal polynomial $m(x)=m_{T}(x)=\prod_{i=1}^{r} p_{i}(x)^{d_{i}}$

ii) the primary partition $\operatorname{dim} \mathbb{V}=\sum_{i=1}^{r} \operatorname{dim} \mathbb{V}_{i}$ where $\mathbb{V}_{i}=\operatorname{ker} p_{i}(T)^{d_{i}}$, and

iii) the secondary partitions with $s_{i, j}$ 's being the exponents in the elementary

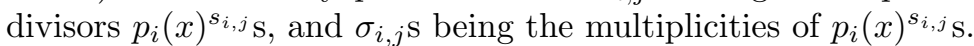

Conversely suppose we have the data i), ii), iii). We first show that there actually exists $T$ in $L(\mathbb{V})$ which realizes this data, and secondly that any two elements in $L(\mathbb{V})$ having the same data are in the same $G L(\mathbb{V})$-orbit.

Take an arbitrary decomposition $\mathbb{V}=\oplus_{i=1}^{r} \mathbb{V}_{i}$ patterned over the primary partition. Next construct an appropriate flag in each $\mathbb{V}_{i}$ with type given by the pairs $\left(s_{i, j}, \sigma_{i, j}\right)$ 's. Let $\mathbb{E}_{i}=\mathbb{F}[x] /\left(p_{i}(x)\right)$, and $\alpha=[x]$. Equip the sub-quotients in the flag in $\mathbb{V}_{i}$ with a compatible family of $\mathbb{E}_{i}$-structures. Take an arbitrary $\mathbb{E}_{i}$-basis $\left(e_{1}, e_{2}, \ldots, e_{k}\right)$ in the component $\mathbb{V}_{0,1}$ of the flag. (We have actually $\left.k=\sigma_{s_{r}}.\right)$ Then 


$$
\left(e_{1}, \alpha e_{1}, \alpha^{2} e_{1}, \ldots, \alpha^{m_{i}-1} e_{1}, e_{2}, \alpha e_{2}, \ldots \ldots, \alpha^{m_{i}-1} e_{k}\right)
$$

is an $\mathbb{F}$-basis of $\mathbb{V}_{0,1}$. Moreover we can define the operator $T$ on $\mathbb{V}_{0,1}$ which is multiplication by $\alpha$. We can continue this process to all the components in the chain ending in $\mathbb{V}_{1}$, and define the operator $T$ on $\mathbb{V}_{1}$ having the minimal polynomial $p(x)$. Next we consider the component $\mathbb{V}_{1,1}$ in the flag. Notice that by construction $\operatorname{dim}_{\mathbb{F}} \mathbb{V}_{1,1} / \mathbb{V}_{1}$ is $m_{i} k$, and $\mathbb{V}_{1,1} / \mathbb{V}_{1}$ has an $\mathbb{E}_{i}$-structure. Choose $\left(e_{1}^{\prime}, e_{2}^{\prime}, \ldots, e_{k}^{\prime}\right)$ in $\mathbb{V}_{1,1}$ whose classes $\left[e_{i}^{\prime}\right]$ modulo $\mathbb{V}_{1}$ form an $\mathbb{E}_{i}$-basis. Define $T^{j} e_{u}^{\prime}, 1 \leq j \leq m-1,1 \leq u \leq k$ in $\mathbb{V}_{1,1}$ so that their classes $\left[T^{j} e_{u}^{\prime}\right]$ modulo $\mathbb{V}_{1}$ are $\left[\alpha^{j} e_{u}\right]$. Now a crucial point is to define $p(T) e_{i}^{\prime}=e_{i}$ in $\mathbb{V}_{0,1}$, and more generally $p(T) T^{j} e_{i}^{\prime}=T^{j} e_{i}, 1 \leq j \leq m_{i}-1$. It is easy to see that continuing this process along the successive components in the flag we obtain a basis of $\mathbb{V}_{i}$ and an operator $T$ in $L\left(\mathbb{V}_{i}\right)$ having the given secondary partition on $\mathbb{V}_{i}$. Taking the direct sum we obtain an operator $T$ on $\mathbb{V}$ having the minimal polynomial $m(x)$ and the given primary and secondary partitions.

Finally suppose that $T, T^{\prime}$ are two elements in $L(\mathbb{V})$ having the same data. Then the dimension of a primary component $\mathbb{V}_{i}$ equals $m_{i} l_{i}$. (Here $l_{i}$ is the largest power of $p_{i}(x)$ dividing the characteristic polynomial.) So by appropriate conjugation by an element of $G L(\mathbb{V})$ we may suppose that both $T$ and $T^{\prime}$ have the same primary components $\mathbb{V}_{i}$ s. So we reduce to the case where $m_{T}(x)=m_{T^{\prime}}(x)=p(x)^{d}$, where $p(x)$ is a monic irreducible in $\mathbb{F}[x]$. Next by hypothesis $T, T^{\prime}$ have the same secondary partitions. Then we can construct the flags and the bases $e_{j}$ 's, $e_{j}^{\prime}$ 's of $\mathbb{V}$ adapted to the respective flags. Then the element $g \in G L(\mathbb{V}), g e_{i} \mapsto e_{i}^{\prime}$ conjugates $T$ into $T^{\prime}$.

This finishes the proof of part A) of theorem 7.1. The proof of part B) can be completed along the same lines using the results in section 6 .

As for the proof of Theorem 7.3, observe that the data the isomorphism class of $(\mathbb{E}, \alpha)$ determines an irreducible polynomial in $\mathbb{F}[x]$. So the proof may be completed along the lines of theorem 7.1.

9. Proof of the Parametrization Theorem 7.2. Let $S, T$ be in the same $z$-class in $L(\mathbb{V})$. This means that $Z_{L}(S)^{*}$ and $Z_{L}(T)^{*}$ are conjugate by an element $u$ in $G L(\mathbb{V})$. First we show that this implies that $Z_{L}(S)$ and $Z_{L}(T)$ are conjugate, in fact by the same element $u$, in $G L(\mathbb{V})$. This follows from the following lemma.

LEMMA 9.1. Let $T$ be in $L(\mathbb{V})$. Then $Z_{L}(T)$ as an $\mathbb{F}$-subalgebra of $L(\mathbb{V})$ and $Z_{L}(T)^{*}$ as a subgroup of $G L(\mathbb{V})$ uniquely determine each other.

Proof. Indeed $Z_{L}(T)$ determines $Z_{L}(T)^{*}$ as the multiplicative subgroup of its units. Conversely let $S$ be a non-invertible element in $Z_{L}(T)$. Then $m_{S}(x)=x^{k} f(x)$, with $k>0$, and $f(0) \neq 0$. Let $\mathbb{V}_{0}=\operatorname{ker} S^{k}$, and $\mathbb{V}_{1}=\operatorname{ker} f(S)$. So $\mathbb{V}=\mathbb{V}_{0} \oplus \mathbb{V}_{1}$ is a $T$-invariant decomposition. For any such decomposition, let $J_{\mathbb{V}_{0}, \mathbb{V}_{1}}$ denote the operator which is identity on $\mathbb{V}_{0}$, and zero on $\mathbb{V}_{1}$. Then $J_{\mathbb{V}_{0}, \mathbb{V}_{1}}$ is in $Z_{L}(T)$, and $S_{1}=S+J_{\mathbb{V}_{0}, \mathbb{V}_{1}}$ is clearly in $Z_{L}(T)^{*}$. Thus $Z_{L}(T)$ is a linear span of $Z_{L}(T)^{*}$ and the operators $J_{\mathbb{V}_{0}, \mathbb{V}_{1}}$ corresponding to all $T$-invariant decompositions $\mathbb{V}=\mathbb{V}_{0} \oplus \mathbb{V}_{1}$. This proves that $Z_{L}(T)^{*}$ determines $Z_{L}(T)$. $\square$

REMARK 9.2. Although the following observation is not needed in the proof that follows, the above lemma raises a question whether $Z_{L}(T)$ itself is always a linear span of $Z_{L}(T)^{*}$. This is indeed the case if $\mathbb{F}$ has more than two elements. For indeed, let $S, \mathbb{V}_{0}$, and $\mathbb{V}_{1}$ be as in the above proof. Let $c$ be an element in $\mathbb{F}$ different from 0 and 1. Define $U_{1}$ as $\left.(S-I)\right|_{\mathbb{V}_{0}}$ on $\mathbb{V}_{0}$, and $\left.c S\right|_{\mathbb{V}_{1}}$ on $\mathbb{V}_{1}$. Define $U_{2}$ as $\left.I\right|_{\mathbb{V}_{0}}$ on $\mathbb{V}_{0}$, 
and $\left.(1-c) S\right|_{\mathbb{V}_{1}}$ on $\mathbb{V}_{1}$. Then $U_{1}, U_{2}$ are in $Z_{L}(T)^{*}$ and $S=U_{1}+U_{2}$. Thus in fact an element in $Z_{L}(T)$ is a sum of at most two elements in $Z_{L}(T) *$.

REMARK 9.3. The restriction that $\mathbb{F}$ has more than two elements in the above remark is a genuine one. For example consider an $n$-dimensional vector space $\mathbb{V}$ over $\mathbb{F}_{2}$, the field with two elements. Assume $n \geq 2$. Let $T$ be an operator with $m_{T}(x)=$ $x^{k}(x-1)^{l}$, where $k \geq 1, l \geq 1$. Consider the $T$-invariant decomposition $\mathbb{V}=\mathbb{V}_{0} \oplus \mathbb{V}_{1}$, where $\mathbb{V}_{0}=\operatorname{ker} T^{k}$, and $\mathbb{V}_{1}=\operatorname{ker}(T-I)^{l}$. Then $Z_{L}(T)=Z_{L}\left(\left.T\right|_{\mathbb{V}_{0}}\right) \times Z_{L}\left(\left.T\right|_{\mathbb{V}_{1}}\right)$. Clearly $Z_{L}\left(\left.T\right|_{\mathbb{V}_{0}}\right)=\mathbb{F}\left[\left.T\right|_{\mathbb{V}_{0}}\right]$, and $Z_{L}\left(\left.T\right|_{\mathbb{V}_{1}}\right)=\mathbb{F}\left[\left.T\right|_{\mathbb{V}_{1}}\right]$, whereas $Z_{L}(T)^{*}$ consists of $f(T)$ where $f(0)=1$. If we take the sum of even number of elements of $Z_{L}(T)^{*}$ then we get an operator all of whose eigenvalues are 0 . On the other hand if we take the sum of odd number of elements of $Z_{L}(T)^{*}$ then we get an operator all of whose eigenvalues are 1. It follows $T$ cannot be written as a sum of elements of $Z_{L}(T)^{*}$.

In view of the lemma we can assume that $Z_{L}(S)$ and $Z_{L}(T)$ are conjugate by an element $u$ in $G L(\mathbb{V})$. Replacing $S$ by $u S u^{-1}$ we may assume that $Z_{L}(S)=Z_{L}(T)$

Let $C$ be the center of $Z_{L}(T)$. By the Frobenius' bicommutant theorem, we have $C=\mathbb{F}[S]=\mathbb{F}[T]$. It is important to note that $C$ does not determine $T$. However every element of $C$ leaves every $T$-invariant (or $S$-invariant) subspace invariant. Let $p_{i}(x)$ be the primes associated to $T$, and $\mathbb{V}=\oplus \mathbb{V}_{i}$ the corresponding primary decomposition. Let $\mathbb{W}$ be a $T$-invariant subspace of $\mathbb{V}_{i}$ such that the pair ( $\mathbb{W},\left.T\right|_{\mathbb{W}}$ ) is dynamically equivalent to $\left(\mathbb{F}[x] /\left(p_{i}(x)^{d}\right), \mu_{x}\right)$. Then $\mathbb{W}_{j}=\operatorname{ker} p_{i}(x)^{j}, 0 \leq j \leq d$, are precisely all the $T$-invariant subspaces of $\mathbb{W}$. Since a subspace of $\mathbb{V}$ is $T$-invariant iff it is $S$-invariant, it follows that $\mathbb{W}_{j}$ 's are precisely also all the $S$-invariant subspaces of $\mathbb{W}$. It follows that $m_{\left.S\right|_{\mathrm{W}}}(x)$ must be of the form $q(x)^{e}$ where $q(x)$ is a monic irreducible polynomial in $\mathbb{F}(x)$.

Next note that the same $q(x)$ works for every $T$-invariant subspace $\mathbb{U}$ such that the pair $\left(\mathbb{W},\left.T\right|_{\mathbb{W}}\right)$ is dynamically equivalent to $\left(\mathbb{F}[x] /\left(p_{i}(x)^{e}\right), \mu_{x}\right)$ for some $e$. For there exists an operator $A$ in $Z_{L}(T)=Z_{L}(S)$ which maps $\mathbb{W}$ onto $\mathbb{U}$ equivariantly with the action of $S$. It follows that $\mathbb{V}=\oplus \mathbb{V}_{i}$ is also a primary decomposition with respect to $S$. So in particular, $n=\sum_{i} n_{i}, \operatorname{dim} \mathbb{V}_{i}=n_{i}$ is a well-defined choice of a primary partition of $n$. Now restrict the action of $Z_{L}(S)=Z_{L}(T)$ to $\mathbb{V}_{i}$. For the same reason we see that the refined flag, and in particular the secondary partitions are well-defined invariants of $Z_{L}(S)=Z_{L}(T)$, which are independent of the choices of a $T$ with the property $C=\mathbb{F}[T]$. Finally considering the action of $Z_{L}(S)=Z_{L}(T)$ on $\mathbb{V}_{d_{i}} / \mathbb{V}_{d_{i-1}}$ we see that the simple field extension $\mathbb{E}_{i}=\mathbb{F}[x] /\left(p_{i}(x)\right)$ is a well-defined invariant of $Z_{L}(S)=Z_{L}(T)$.

Conversely, given the the primary and secondary partitions and the field extensions $\mathbb{E}_{i}$ 's of appropriate degree there clearly exists an operator having this data, and its orbit class is uniquely determined. This finishes the proof of the theorem $7.2 \mathrm{in}$ the linear case. Using the results of section 6 , the proof can be extended to the affine case.

10. Generating Functions for $z$-classes. Let $\mathcal{D}$ be a collection of extension fields of finite degree of a field $\mathbb{F}$ with the property that $\mathcal{D}$ contains only finitely many extensions of a given degree. Significantly, this property is automatically satisfied for the collection of all extension fields in the following cases: 1) $\mathbb{F}$ algebraically closed, $2) \mathbb{F}=\mathbb{R}, 3) \mathbb{F}=$ a local field, 4) $\mathbb{F}=$ a finite field. A case of arithmetic interest is 5) $\mathbb{F}=\mathbb{Q}, S=$ a finite set of primes, and $\mathcal{D}=$ the collection of all extension fields obtained by adjoining all $n$-th roots of all primes in $S$. From the parametrization 
theorem 7.2, it follows that for any such collection $\mathcal{D}$, and for a fixed $n$, there are only finitely many $z$-classes of linear maps on an $n$-dimensional vector space over $\mathbb{F}$ with the extension fields in $\mathcal{D}$. So one can form a generating function

$$
\mathcal{Z}_{\mathbb{F}, \mathcal{D}}(x)=\Sigma_{n=0}^{\infty} z(n) x^{n} .
$$

As is expected from the parametrization theorems, these functions are closely related to the generating functions for partitions. One may also consider the restricted generating functions which enumerate the $z$-classes of dynamically semi-simple operators, or cyclic operators. In both cases the secondary partitions have simple types. (Recall that a pair $(\mathbb{V}, T)$ is cyclic if there exists a vector $v$ such that $V=\mathbb{F}[T] v$. Clearly $(\mathbb{V}, T)$ is cyclic iff $\operatorname{deg} m_{T}(x)=\operatorname{dim} \mathbb{V}$.) We denote the corresponding generating functions by

$$
\mathcal{Z}_{\mathbb{F}, \mathcal{D}, s}(x) \text { and } \mathcal{Z}_{\mathbb{F}, \mathcal{D}, c}(x)
$$

respectively.

Let $\Pi_{n}$ denote the set of all partitions of $n$, and $p(n)$ the cardinality of $\Pi_{n}$. A partition $\pi$ of $n$ with signature $\left(1^{a_{1}} 2^{a_{2}} \ldots n^{a_{n}}\right)$ is the partition in which $i$ occurs $a_{i}$ times, so $n=\Sigma_{i} a_{i} i$.

Let $f(x)=1+\Sigma_{n=1}^{\infty} b(n) x^{n}$ be a formal power series. To $f(x)$ we associate a new power series, $\mathcal{P}(f(x))=1+\Sigma_{n=1}^{\infty} c(n) x^{n}$. Here $c(n)$ is a sum $\Sigma_{\pi \in \Pi_{n}} c_{\pi}$, where $c_{\pi}=$ $\Pi_{i=1}^{n} b(i)^{a_{i}}$, if $\pi$ has signature $\left(1^{a_{1}} 2^{a_{2}} \ldots n^{a_{n}}\right)$. Notice that the well-known Eulerian generating function for partitions $P(x)=1+\sum_{n=1}^{\infty} p(n) x^{n}$ is $\mathcal{P}(g(x))$ where $g(x)=$ $\Sigma_{n=0}^{\infty} x^{n}$ is the geometric series.

The Absolute Case: Here $\mathbb{F}$ is algebraically closed. Here $\mathcal{D}$ consists of a single element, namely $\mathbb{F}$ itself, and we omit its mention. First consider the easy cases of i) semisimple operators, or ii) cyclic operators. In both cases, the secondary partitions are uniquely determined. Let the primary partition of an operator $T$ be $\pi: n=$ $\sum_{i=1}^{r} n_{i}$. If $T$ is semisimple then the secondary partitions of $n_{i}$ s have signatures $\left(1^{n_{i}}\right)$. If $T$ is cyclic then the secondary partitions of $n_{i}$ s have signatures $\left(n_{i}^{1}\right)$. So

$$
\mathcal{Z}_{s}(x)=\mathcal{Z}_{c}(x)=P(x) .
$$

On the other hand consider the case of all $z$-classes. Let $T$ be the operator whose primary partition has signature $\left(1^{a_{1}} 2^{a_{2}} \ldots n^{a_{n}}\right)$. Then the number of secondary partitions associated with this partition is $p(1)^{a_{1}} p(2)^{a_{2}} \ldots p(n)^{a_{n}}$. It follows that

$$
\mathcal{Z}(x)=\mathcal{P}(P(x))=\Pi_{k=1}^{\infty} \frac{1}{1-p(k) x^{k}} .
$$

THEOREM 10.1. $\mathcal{Z}(x)$ is a meromorphic function on the unit disc, and it cannot be extended beyond the unit disc.

Proof. A simple estimate for $p(n)$ is $p(n) \leq e^{K \sqrt{n}}$, for $K>0$, cf. [3], ch. VII, section 3. It follows that $p(n)^{\frac{1}{n}}$ tends to 1 as $n$ tends to infinity. So for $|x|<1, Z(x)$ defines a meromorphic function on the unit disc. Its poles are at $x=p(n)^{-\frac{1}{n}} e^{\frac{2 k \pi i}{n}}$, for $n=1,2, \ldots$ and $0 \leq k \leq n$. So it also follows that the function cannot be extended meromorphically beyond the unit disc. 
It appears that this type of generating function has not appeared in number theory before.

Notice that if we consider more generally the case of an arbitrary field $\mathbb{F}$, but restrict to $\mathcal{D}=\{\mathbb{F}\}$, then we get the same generating functions.

General Case: Let $\mathbb{E}$ be an element of $\mathcal{D}$, and $[\mathbb{E} ; \mathbb{F}]=m$. Clearly the contribution to $\mathcal{Z}_{\mathcal{D}}(x)$ coming from $\mathbb{E}$ is $\mathcal{Z}\left(x^{m}\right)$. We denote this contribution by $\mathcal{Z}_{\mathbb{F}, \mathbb{E}}(x)$. Clearly

$$
\mathcal{Z}_{\mathbb{F}, \mathcal{D}}(x)=\Pi_{\mathbb{E} \in \mathcal{D}} \mathcal{Z}_{\mathbb{F}, \mathbb{E}}(x) .
$$

Since we have assumed that $\mathcal{D}$ contains only finitely many extensions of a given degree, this product is well-defined. Two notable cases are i) $\mathbb{F}=\mathbb{R}$, the field of real numbers, and ii) $\mathbb{F}=\mathbb{F}_{q}$, the finite field with $q$ elements, and $\mathcal{D}$ consists of all extensions of finite degree. Then

$$
\begin{gathered}
\mathcal{Z}_{\mathbb{R}, \mathcal{D}}(x)=\mathcal{Z}(x) \mathcal{Z}\left(x^{2}\right) . \\
\mathcal{Z}_{\mathbb{F}_{q}, \mathcal{D}}(x)=\Pi_{n=1}^{\infty} \mathcal{Z}\left(x^{n}\right) .
\end{gathered}
$$

\section{REFERENCES}

[1] A. Borel, Linear algebraic groups, second edition. Graduate Texts in Mathematics, 126. Springer-Verlag, New York, 1991. xii+288 pp. ISBN: 0-387-97370-2.

[2] A. Borel, The work of Chevalley in Lie groups and algebraic groups, Proceedings of the Hyderabad Conference on Algebraic Groups (Hyderabad, 1989), 1-22, Manoj Prakashan, Madras, 1991.

[3] K. Chandrasekharan, Arithmetic Functions, Die Grundlehren der mathematischen Wissenschaften in Einzeldarstellungen, 167, Springer Verlag, 1970.

[4] C. Chevalley, A new kind of relationship between matrices, Amer. J. Math., 65 (1943), pp. $521-531$.

[5] E. A. Coddington and N. Levinson, Theory of ordinary differential equations, McGraw-Hill Book Company, Inc., New York-Toronto-London, 1955. xii+429 pp.

[6] R. Gouraige, z-Classes of Elements in Central Simple Algebras, thesis, City University of New York (2006).

[7] M. W. Hirsch And S. Smale, Differential equations, dynamical systems, and linear algebra, Pure and Applied Mathematics, Vol. 60. Academic Press [A subsidiary of Harcourt Brace Jovanovich, Publishers], New York-London, 1974. xi+358 pp.

[8] N. Jacobson, Basic algebra, I. Second edition. W. H. Freeman and Company, New York, 1985. xviii+499 pp. ISBN: 0-7167-1480-9.

[9] R. S. Kulkarni, Dynamical Types, and Conjugacy Classes of Centralizers in Groups, J. Ramanujan Math. Soc., 22 (2007), pp. 35-56.

[10] G. PILZ, Near-rings, the theory and its applications, North-Holland Mathematics Studies, No. 23. North-Holland Publishing Co., Amsterdam-New York-Oxford, 1977. xiv+393 pp.

[11] J. H. M. Wedderburn, The canonical form of a matrix, Ann. of Math. (2), 39 (1938), pp. 178180 . 
R. S. KULKARNI 